\title{
Attitude Estimation for In-Service Base Station Antenna Using Downlink Channel Fading Statistics
}

\author{
Cen Ling, ${ }^{1}$ Xuefeng Yin, ${ }^{1}$ Yongyu He, ${ }^{1}$ and Silvia Ruiz Boquée ${ }^{2}$ \\ ${ }^{1}$ School of Electronics and Information Engineering, Tongii University, Telecom Building, 4800 Caoan Road, Shanghai 201804, China \\ ${ }^{2}$ Department of Signal Theory and Communications, Polytechnic University of Catalonia, Building C4, C. Esteve Terradas 7 , \\ 08860 Castelldefels, Spain
}

Correspondence should be addressed to Xuefeng Yin; yinxuefeng@tongji.edu.cn

Received 24 November 2014; Revised 18 February 2015; Accepted 3 March 2015

Academic Editor: Welsy Wai-Wa Choi

Copyright (C) 2015 Cen Ling et al. This is an open access article distributed under the Creative Commons Attribution License, which permits unrestricted use, distribution, and reproduction in any medium, provided the original work is properly cited.

\begin{abstract}
A maximum-likelihood-estimation method is proposed for extracting the attitude of a sectoring base station (BS) antenna by using the received signal strengths observed by multiple user equipments (UEs) in this contribution. This method calculates the likelihood function of the antenna attitude derived by taking into account the multiscale fading statistics, that is, path loss, shadowing, and multipath fading. Depending on whether a calibration result of these fading statistics is available or not, the proposed method can be utilized in either calibration-based estimation (CBE) or calibration-free estimation (CFE) approaches. The performance of both methods is evaluated by Monte-Carlo simulations and real experiments. The results obtained demonstrate that the estimation accuracy of both CBE and CFE approaches increases when the percentage of UEs in the line-of-sight (LoS) condition among all available UEs increases and, moreover, the total number of UEs has no significant impact on the estimation accuracy. Furthermore, the CFE exhibits more robust performance than the CBE particularly in the case where the calibration results involve uncertainties.
\end{abstract}

\section{Introduction}

For cellular communication networks, the intercell interference (ICI) is one of the main causes of deterioration of network coverage, system capacity, and traffic throughput [13]. Beamforming and adaptive antenna techniques have been adopted at the base stations (BSs) to alleviate the ICI [4]. In addition, selecting appropriately the operational parameters of the BSs in a network, that is, the antenna height, antenna tilting, and pilot power, is also effective in improving cell isolation and optimizing the coverage of the cellular system [5].

The attitude of a BS antenna is represented by the antenna tilted elevation and azimuth. The former is defined as the angle between the direction of the main beam of the antenna and the horizontal plane. Positive and negative tilted elevation are usually called as downtilt and uptilt, respectively. Properly setting the antenna downtilt and uptilt can help reduce the ICI and, meanwhile, enhance the coverage in the local area. The antenna azimuth, defined empirically as the azimuth of the direction of the antenna's main lobe with $0^{\circ}$ azimuth referred to as the due North, is another configuration parameter yielding a significant effect on network coverage and cell overlap [6]. Adaptation of antenna attitude can be performed mechanically, electrically, or by a combination of both approaches [7-10].

Recently, researches on adapting BS antenna attitude for optimizing system performance have advanced quickly due to its promising usage in cooperative communication systems and in the self-organizing networks (SONs) with heterogeneous BS and user equipments (UEs) [11, 12]. The classical "antenna tilting techniques (ATTs)" [13, 14] can be used to direct a coverage notch intentionally to the center of horizontal beam pattern of a cochannel BS antenna, based on the principle of ICI mitigation for both interior UEs and those located at cell edges $[15,16]$. ATTs can also be applied to mitigate congestion in hot-spot sectors when the traffic is nonuniformly distributed $[17,18]$. The optimal downtilt can be determined by using a function of site spacing, antenna height, and vertical beamwidth $[19,20]$ or based on the wave propagation direction and the polarization of the signals [21].

ATTs are usually performed under the assumption that the original antenna attitude is known in advance. This is true 
for the conventional communication networks consisting of macro- or microcellular BSs. However, as the network architecture becomes more heterogenous, smaller BSs either fixed or nomadic would be widely deployed, for example, in the fourth and fifth generation communication systems [22]. In such cases, a BS may be positioned unexpectedly in such a way that the antenna attitude is different from the predefined status significantly. Thus, estimation of antenna attitude is a necessity for applying ATTs. As far as we are concerned, the techniques for antenna attitude estimation available in literature are either only applicable under the line-of-sight (LoS) connection assumption in, for example, synthetic aperture radar applications [23] or using oversimplified approaches without considering the impact of multipath propagation [24]. These methods lead to poor performance when being applied in cellular communication environments where multiple UEs exist with significant mobilities in either LoS or non-line-of-sight (NLoS) conditions.

To fill the aforementioned gap, we propose in this manuscript a novel approach of estimating the BS antenna attitude in realistic cellular communication scenarios, based on the received signal strength reported by multiple UEs under the coverage of the BS. According to fading mechanisms, the observed signal power is decomposed into multiple variables following different probability density functions (pdfs). Depending on whether a calibration result of these pdfs is available or not, the proposed method can be utilized in either calibration-based estimation (CBE) or calibration-free estimation (CFE) approaches. The CBE makes use of the path loss models and the statistics of shadowing and multipath fading predefined for specific types of environments, while the CFE relies on only the assumption that the fading in different scales follows certain types of distributions. The performances of $\mathrm{CBE}$ and $\mathrm{CFE}$ are investigated by both Monte-carlo simulations and on-field measurements. The major contributions of our work reported here summarized are as follows. (i) The proposed methodology is the first of its kind of adopting the maximum likelihood (ML) principle for estimating the BS antenna attitude; (ii) different from the conventional methods which were assessed only by simulations, our methods are proved to be effective by an extensive measurement campaign in realistic cellular environments.

The rest of the paper proceeds as follows. In Section 2, the model of the received signal power in the UE is introduced. In Section 3, the maximum-likelihood-estimation for the attitude of the BS antenna is presented. Simulation results are elaborated in Section 4 for the performance assessment of the proposed method. Measurement-based investigation results are presented in Section 5. Eventually, conclusive remarks are given in Section 6.

\section{The Model of Channel Gain}

Let us consider a cellular communication scenario in an outdoor environment where the UEs are distributed in the coverage of a BS. The signal power transmitted by the BS is denoted by $P_{t}$. The normal direction of the BS antenna is tilted by the direction $\eta_{\mathrm{Tx}}=\left(\theta_{\mathrm{Tx}}, \varphi_{\mathrm{Tx}}\right)$, where the subscript "Tx" refers to the transmitter which is the BS in our case;
$\theta_{\mathrm{Tx}}$ and $\varphi_{\mathrm{Tx}}$ are the elevation tilting angle, that is, downtilt and azimuth angle of the BS antenna, respectively. In this contribution, we are interested in estimating $\eta_{\mathrm{Tx}}$ which is unknown due to some reasons, for example, mechanical errors. The UEs are assumed to be located in clutter environments where the scattering caused by the objects in the UE's vicinity is the major source for wave propagation. A UE antenna usually has a large half-power beamwidth for its main lobe. In addition, the impact of the user's hand holding the mobile phone on the radiation pattern of the phone antenna results in the near-omnidirectional radiation pattern $[25,26]$. Therefore, for simplicity, in our study the UE's antenna is considered to have omnidirectional radiation pattern and hence the attitude of UE is irrelevant in the investigation.

The gain of the radio channel experienced by signals received by a UE can be factorized as

$$
g\left(\boldsymbol{\Omega}_{\mathrm{Tx}}, d ; \eta_{\mathrm{Tx}}\right)=g_{p}(d) \cdot g_{s} \cdot g_{m} \cdot g_{\mathrm{Tx}}\left(\boldsymbol{\Omega}_{\mathrm{Tx}} ; \eta_{\mathrm{Tx}}\right) \cdot g_{\mathrm{Rx}},
$$

where the subscript " $\mathrm{Rx}$ " refers to the receiver which is the $\mathrm{UE}$ in the case considered here, $d$ is the distance represented in meter between the BS and the UE, $g_{p}(d), g_{s}$, and $g_{m}$ denote the gain due to the free-space propagation influenced by the terrain, the shadow fading, and multipath fading, respectively, $g_{\mathrm{Tx}}\left(\boldsymbol{\Omega}_{\mathrm{Tx}} ; \eta_{\mathrm{Tx}}\right)$ is the BS antenna gain at the direction of departure (DoD), that is, $\boldsymbol{\Omega}_{\mathrm{Tx}}$ when the antenna is titled by $\eta_{\mathrm{Tx}}$, and finally $g_{\mathrm{Rx}}$ is the UE antenna gain. Here, the assumption that the propagation towards the UE is characterized by the same DoD is applied. This is reasonable in our case where the objects interacting with the waves are located in the UEs' vicinity in such a way that the DoD dispersion of a downlink channel is so concentrated that the BS antenna gains added to all paths impinging at the UE antenna can be approximated by an identical BS antenna gain $g_{\mathrm{Tx}}\left(\boldsymbol{\Omega}_{\mathrm{Tx}} ; \eta_{\mathrm{Tx}}\right)$.

The assumptions adopted here for analyzing channels include the following.

(1) The average path loss in large-scale for an arbitrary $\mathrm{Tx}-\mathrm{Rx}$ separation can be expressed as a function of distance:

$$
\overline{\mathrm{PL}}(d)_{\mathrm{dB}}=\overline{\mathrm{PL}}\left(d_{0}\right)+10 n \cdot \log _{10}\left(\frac{d}{d_{0}}\right),
$$

where $n$ is the path loss exponent (PLE) which indicates the rate at which the path loss increases with distance, $d_{0}$ is the close-in reference distance, which is $d_{0}=1 \mathrm{~m}$, and $d$ is the T-R separation distance [3].

(2) The shadow-fading gain $g_{s}$ follows a lognormal (LN) distribution with zero mean and standard deviation $\sigma_{s}[27-29]$ :

$$
f_{\mathrm{LN}}\left(g_{s}\right)=\frac{1}{\sqrt{2 \pi} \sigma_{s} g_{s}} \exp \left\{-\frac{\ln \left(g_{s}\right)^{2}}{2 \sigma_{s}^{2}}\right\} \text {. }
$$

(3) The multipath fading $g_{m}$ is uncorrelated with $g_{s}$ and follows the Rician ( $\mathrm{Ri}$ ) distribution with mean value $\mu_{m}$ and scaling parameter $\sigma_{m}[3,30]$ :

$$
f_{\mathrm{Ri}}\left(g_{m}\right)=\frac{\sqrt{g_{m}}}{\sigma_{m}^{2}} \exp \left\{-\frac{g_{m}+\mu_{m}^{2}}{2 \sigma_{m}^{2}}\right\} I_{0}\left\{\frac{\mu_{m} \sqrt{g_{m}}}{\sigma_{m}^{2}}\right\} \text {, }
$$


where $I_{0}(\cdot)$ denotes the zero-order modified Bessel function of the first kind. Notice that the Rayleigh distribution is a special case of the Rician distribution with $\mu_{m}=0$.

(4) The BS antenna gain $g_{\mathrm{Tx}}\left(\Omega_{\mathrm{Tx}} ; \eta_{\mathrm{Tx}}\right)$ is deterministic.

(5) The UE antenna gain $g_{\mathrm{Rx}}$ is identical for all UEs.

Since $g_{p}(d), g_{s}, g_{m}, g_{\mathrm{Tx}}\left(\Omega_{\mathrm{Tx}} ; \eta_{\mathrm{Tx}}\right)$, and $g_{\mathrm{Rx}}$ are uncorrelated, the joint probability of multiple observations $\mathbf{g}=$ $\left\{g\left(\Omega_{\mathrm{Tx}}, d\right) ; \Omega_{\mathrm{Tx}} \in S_{\mathrm{Tx}}, d \in\left[d_{\min }, d_{\max }\right]\right\}$ with $\mathcal{S}_{\mathrm{Tx}}$ being a unit sphere centered at the Tx and $d_{\min }, d_{\max }$ denoting the minimum and maximum separation between the BS and UEs, respectively, can be written as

$$
\begin{aligned}
f\left(\mathbf{g} ; \eta_{\mathrm{Tx}}\right)= & \prod_{\boldsymbol{\Omega}_{\mathrm{Tx}}, d} f\left(g\left(\boldsymbol{\Omega}_{\mathrm{Tx}}, d ; \eta_{\mathrm{Tx}}\right)\right) \\
= & \prod_{\boldsymbol{\Omega}_{\mathrm{Tx}}, d} f\left(g_{p}\left(\boldsymbol{\Omega}_{\mathrm{Tx}}, d ; \eta_{\mathrm{Tx}}\right)\right) f\left(g_{s}\left(\boldsymbol{\Omega}_{\mathrm{Tx}}, d ; \eta_{\mathrm{Tx}}\right)\right) \\
& \cdot f\left(g_{m}\left(\boldsymbol{\Omega}_{\mathrm{Tx}}, d ; \eta_{\mathrm{Tx}}\right)\right) f\left(g_{\mathrm{Tx}}\left(\boldsymbol{\Omega}_{\mathrm{Rx}}, d ; \eta_{\mathrm{Tx}}\right)\right) \\
& \cdot f\left(g_{\mathrm{Rx}}\right) .
\end{aligned}
$$

Considering that the average path loss and the BS and UE antenna gains are all deterministic, (5) can be rewritten as

$$
\begin{aligned}
f\left(\mathbf{g} ; \eta_{\mathrm{Tx}}\right) & \\
& \propto \prod_{\boldsymbol{\Omega}_{\mathrm{Tx}}, d} f\left(g_{s}\left(\boldsymbol{\Omega}_{\mathrm{Tx}}, d ; \eta_{\mathrm{Tx}}\right)\right) f\left(g_{m}\left(\boldsymbol{\Omega}_{\mathrm{Tx}}, d ; \eta_{\mathrm{Tx}}\right)\right) \\
& =\prod_{\boldsymbol{\Omega}_{\mathrm{Tx}}, d} f_{\mathrm{LN}}\left(g_{s}\left(\boldsymbol{\Omega}_{\mathrm{Tx}}, d ; \eta_{\mathrm{Tx}}\right)\right) f_{\mathrm{Ri}}\left(g_{m}\left(\boldsymbol{\Omega}_{\mathrm{Tx}}, d ; \eta_{\mathrm{Tx}}\right)\right) .
\end{aligned}
$$

Here, $g_{s}\left(\Omega_{\mathrm{Tx}}, d ; \eta_{\mathrm{Tx}}\right)$ is obtained by averaging multiple values of $g\left(\Omega_{\mathrm{Tx}}, d ; \eta_{\mathrm{Tx}}\right) / g_{p}\left(d ; \eta_{\mathrm{Tx}}\right)$ observed within the range of tens of wavelengths around the UE [27] and $g_{m}\left(\Omega_{\mathrm{Tx}}, d ; \eta_{\mathrm{Tx}}\right)$ is calculated by averaging the values of $g\left(\Omega_{\mathrm{Tx}}, d ; \eta_{\mathrm{Tx}}\right) / g_{p}\left(d ; \eta_{\mathrm{Tx}}\right) / g_{s}\left(\Omega_{\mathrm{Tx}}, d ; \eta_{\mathrm{Tx}}\right)$ observed within the distance of several wavelengths centered at $d$. Notice that the path loss model for $g_{p}(d)$ may have different values for the decay exponent constant and floating intercept depending on the urban/suburban and LoS/NLoS scenarios [31]. Thus, in order to calculate $f\left(\mathbf{g} ; \eta_{\mathrm{Tx}}\right)$ accurately, the path loss $g_{p}\left(d ; \eta_{\mathrm{Tx}}\right)$ for a certain UE need to be calculated by using specific path loss models in accordance with the environment where the BS and the UE are located.

\section{Antenna Attitude Estimation Techniques}

The problem at hand is to estimate the antenna attitude parameter $\eta_{\mathrm{Tx}}$ based on the observations $\mathbf{g}$. As the UEs are randomly distributed across the coverage sector, g, being the observed feedback from the UEs, follows the uniform distribution, and thus $f(\mathbf{g})$ remains constant. From the Bayesian theorem, that is, $f\left(\mathbf{g} ; \eta_{\mathrm{Tx}}\right)=f\left(\eta_{\mathrm{Tx}} ; \mathbf{g}\right) \cdot f(\mathbf{g}) / f\left(\eta_{\mathrm{Tx}}\right)$ with the uniform priori probability of $\eta_{\mathrm{Tx}}$ and fixed $f(\mathbf{g})$, the likelihood of $\eta_{\mathrm{Tx}}$, given $\mathbf{g}$, that is, the posterior probability $f\left(\eta_{\mathrm{Tx}} ; \mathbf{g}\right)$, can be calculated as

$$
f\left(\eta_{\mathrm{Tx}} ; \mathbf{g}\right) \propto f\left(\mathbf{g} ; \eta_{\mathrm{Tx}}\right) .
$$

The ML estimate of $\eta_{\mathrm{Tx}}$ can be obtained by solving the following optimization problem:

$$
\left(\widehat{\eta}_{\mathrm{Tx}}\right)_{\mathrm{ML}}=\arg \max _{\eta_{\mathrm{Tx}}} \Lambda\left(\eta_{\mathrm{Tx}} ; \mathbf{g}, \sigma_{s}, \mu_{m}, \sigma_{m}\right),
$$

where $\Lambda\left(\eta_{\mathrm{Tx}} ; \mathbf{g}, \sigma_{s}, \mu_{m}, \sigma_{m}\right)$ is the objective function. Under the assumption that $\sigma_{s}, \mu_{m}$, and $\sigma_{m}$ are constant for all UEs within the coverage of the BS antenna, the objective function can be calculated, by dropping the constant components as

$$
\begin{aligned}
\Lambda\left(\eta_{\mathrm{Tx}} ; \mathbf{g}, \sigma_{s}, \mu_{m}, \sigma_{m}\right) & \\
=\int_{d_{\min }}^{d_{\max }} \int_{\mathcal{S}_{\mathrm{Tx}}} & -\frac{\ln \left(g_{s}\left(\boldsymbol{\Omega}_{\mathrm{Tx}}, d ; \eta_{\mathrm{Tx}}\right)\right)^{2}}{2 \sigma_{s}^{2}} \\
& -\frac{g_{m}\left(\boldsymbol{\Omega}_{\mathrm{Tx}}, d ; \eta_{\mathrm{Tx}}\right)+\mu_{m}^{2}}{2 \sigma_{m}^{2}} \\
& +\ln I_{0}\left\{\frac{\mu_{m} \sqrt{g_{m}\left(\boldsymbol{\Omega}_{\mathrm{Tx}}, d ; \eta_{\mathrm{Tx}}\right)}}{\sigma_{m}^{2}}\right\} \\
& +\frac{\ln \sqrt{g_{m}\left(\boldsymbol{\Omega}_{\mathrm{Tx}}, d ; \eta_{\mathrm{Tx}}\right)}}{\sqrt{2 \pi} \sigma_{s} g_{s} \sigma_{m}^{2}} \mathrm{~d} \boldsymbol{\Omega}_{\mathrm{Tx}} \mathrm{d} d .
\end{aligned}
$$

In the case where $\sigma_{s}, \mu_{m}$, and $\sigma_{m}$ are dependant on $\boldsymbol{\Omega}_{\mathrm{Tx}}$ and $d$, $\sigma_{s}, \mu_{m}$, and $\sigma_{m}$ should be substituted by their functions with respect to $\left(\boldsymbol{\Omega}_{\mathrm{Tx}}, d\right)$, respectively. For simplicity, the case where $\sigma_{s}, \mu_{m}$, and $\sigma_{m}$ are constant is considered in the sequel. In the CBE method, it is a prerequisite to conduct calibration campaigns to get the correct estimate of the path loss model $g_{p}(d)$ and the model parameters, that is, $\sigma_{s}, \mu_{m}$, and $\sigma_{m}$, in order to calculate the ML estimate of $\eta_{\mathrm{Tx}}$. In the calibration campaigns, the radiation pattern of the BS antenna and its attitude is assumed to be known in advance. Steps for performing the CBE method are described as follows.

(1) The path loss model is established by fitting a regression line to the empirical scatter plots of the averaged power loss expressed in decibel (dB) versus the BSUE separation. The average power loss is calculated by taking the arithmetic mean of the received signal power obtained within the distance of hundreds of wavelengths [27].

(2) Then the shadow fading is calculated by removing the power loss predicted by the path loss model and taking the average of the residue within the distance of tens of wavelengths.

(3) In addition, the multipath fading is obtained by removing both the power loss, predicted by the path loss model, and the shadow fading.

(4) Then the lognormal pdf and the Rician pdf are fitted to the empirical pdfs of the shadow fading and multipath fading, respectively. 
(5) For any fixed candidate attitude $\eta_{\mathrm{Tx}}$, the likelihood of $\Lambda\left(\sigma_{s}, \mu_{m}, \sigma_{m} ; \mathbf{g}, \eta_{\mathrm{Tx}}\right)$, which has the same expression of $\Lambda\left(\eta_{\mathrm{Tx}} ; \mathbf{g}, \sigma_{s}, \mu_{m}, \sigma_{m}\right)$ in (9), is maximized over $\left[\sigma_{s}, \mu_{m} \cdot \sigma_{m}\right]$. Such maximal values are collected and the corresponding $\eta_{\mathrm{Tx}}$ 's are recorded. Then, a specific $\eta_{\mathrm{Tx}}$ that yields the maximum among these values is considered to be the estimate of antenna attitude.

In the CFE method, only the assumption that the gains due to shadow fading and multipath fading follow, respectively, the lognormal and Rician distributions is adopted. No calibration is required in the CFE method and parameters for the specific environment, that is $\left[\sigma_{s}, \mu_{m} \cdot \sigma_{m}\right]$, are not available in advance. At every time, $\left[\sigma_{s}, \mu_{m} \cdot \sigma_{m}\right]$ are, respectively, obtained given the existing signal feedbacks. Steps for calculating the likelihood and estimating of the antenna attitude are the similar to the CBE method.

As mentioned in Section 2, the path loss model can be distinctive depending on the environments where the BS and the UE are located. Considering that the LoS and NLoS scenarios can be accurately distinguished by analyzing the $K$-factor or delay spread of the channel $[32,33]$, it is natural to consider two path loss models applicable for the LoS and NLoS scenarios, respectively. Thus, for calibration campaigns conducted for a specific type of environment, the estimates of the parameters: $\boldsymbol{\kappa}=\left[n_{\mathrm{LoS}}, n_{\mathrm{NLoS}}, \sigma_{s}, \mu_{m} \cdot \sigma_{m}\right]$, are obtained, where $n_{\mathrm{LoS}}$ and $n_{\mathrm{NLoS}}$ represent the path loss exponent constant in the LoS and NLoS scenarios, respectively. Then these parameter estimates are applied in (8) to calculate $\left(\widehat{\eta}_{\mathrm{Tx}}\right)_{\mathrm{ML}}$ when the environment is of the same type as considered in the calibration campaign. It is worth mentioning that the CBE and CFE techniques can be implemented by taking into account the realistic $\mathrm{BS}$ antenna radiation pattern under the settings of different antenna attitude. This makes the techniques applicable for estimating both the mechanical and the electrical tilts in cases where the antenna pattern might be adaptive.

It is noted that signals received from the LoS path are more sensitive to the variation of the BS antenna attitude compared with the NLoS paths. This is reasonable since the signals are attenuated and distorted significantly by the propagation channels in the NLoS scenario, and thus the estimate of the antenna attitude may exhibit larger variance in NLoS than in LoS cases. Therefore, this algorithm for estimating antenna attitude of the BS performs better in cases with larger percentage of LoS users and is more applicable for the scenarios with a multitude of LoS users, such as in the environments of plaza, shopping mall, stadium, and playground.

\section{Numerical Simulations}

Monte-Carlo simulations are performed to evaluate the performance of the CBE and CFE techniques proposed in Section 3. A modified graph modeling method based on the original scheme introduced in [34] is applied to generate the synthetic propagation channels in urban environments. In this section, we briefly introduce the graph modeling adopted in the simulations. Then, the evaluation results for the performance of the $\mathrm{CBE}$ and $\mathrm{CFE}$ techniques are elaborated.

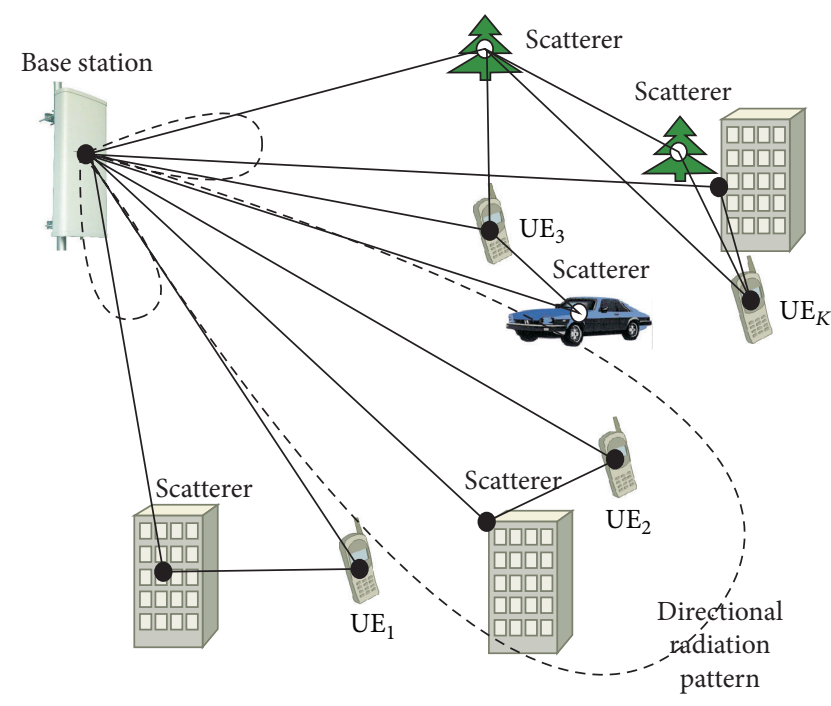

Figure 1: An example of the propagation graph.

4.1. Graph Modeling for Synthetic Channel Generation. The stochastic propagation-graph channel modeling has been introduced for generating random channel realizations in synthetic environments [34-36]. This approach relies on the geometrical positions, the mobility, and electromagnetic properties of scatterers that are distributed in plausible positions in the environments of interests. It emulates the direct propagation of electromagnetic (EM) waves from the Tx to the Rx, from the Tx to the scatterers, from the scatterers to the $\mathrm{Rx}$, and among the scatterers due to the reverberation of EM waves. The channel impulse responses (CIRs) are generated by superimposing the contributions of multiple paths where the number of the bouncing points or hops along a path ranges from 0 to infinity.

Figure 1 illustrates a diagram of propagation graph used in the simulations. The single BS installed with an antenna with a directional radiation pattern emits signals via its outgoing edges to the $K$, UEs, and $N$ scatterers simultaneously in the environment concerned. A direct link may exist between the BS and a UE. A scatterer adds up the signals on its ingoing edges and transmits the result obtained on the outgoing edges with certain weights. The signal collected by a UE is the superposition of the signals arriving via multiple ingoing edges. Propagation along edges with different lengths leads to the delay dispersion of the received signal. This effect can be calculated based on the geometrical positions of the edge ends and the mechanisms of interactions between EM waves and scatterers, such as reflection, diffraction, and scattering. Assuming that the responses of the interactions are linear and time-invariant, the received signal at a UE can be calculated via convolution with the resultant temporal CIR or multiplication with the channel transfer function $\mathbf{H}(f)$ in the frequency domain. In the simulations considered here, $\mathbf{H}(f)$ is calculated by using the graph modeling method modified from that described in [34] as

$$
\begin{aligned}
\mathbf{H}(f)= & \mathbf{D}(f) \odot \mathbf{G}_{\mathrm{LoS}}(f)+\mathbf{R}(f) \\
& \cdot\left[1+\mathbf{B}(f)+\mathbf{B}^{2}(f)+\cdots+\mathbf{B}^{n}(f)+\cdots\right] \\
& \cdot\left[\mathbf{T}(f) \odot \mathbf{G}_{\mathrm{NLoS}}(f)\right]
\end{aligned}
$$




$$
\begin{aligned}
= & \mathbf{D}(f) \odot \mathbf{G}_{\mathrm{LoS}}(f)+\mathbf{R}(f)[1-\mathbf{B}(f)]^{-1} \\
& \cdot\left[\mathbf{T}(f) \odot \mathbf{G}_{\mathrm{NLoS}}(f)\right],
\end{aligned}
$$

where $\mathbf{H}(f) \in \mathscr{C}^{1 \times K}$ contains the coefficients of channels between the BS and totally $K$ UEs, $\mathbf{D}(f) \in \mathscr{C}^{1 \times K}$ is the transfer function for the direct links between the BS and UEs, $\odot$ refers to the element-wise product operation, $\mathbf{G}_{\mathrm{LoS}}(f) \in \mathscr{C}^{1 \times K}$ and $\mathbf{G}_{\mathrm{NLoS}}(f) \in \mathscr{C}^{1 \times N}$ represent the BS antenna gains for the LoS paths and for the paths between the BS and the scatterers, respectively, and $\mathbf{T}(f) \in \mathscr{C}^{1 \times N}, \mathbf{R}(f) \in$ $\mathscr{C}^{K \times N}$, and $\mathbf{B}(f) \in \mathscr{C}^{N \times N}$ denote the transfer functions between the BS and scatterers, the scatterers and UEs, and among the scatterers, respectively. Notice that, in the righthand side of $(10), \mathbf{D}(f) \odot \mathbf{G}_{\mathrm{Los}}(f)$ counts for the contributions of the LoS paths and $\mathbf{R}(f)[1-\mathbf{B}(f)]^{-1}\left(\mathbf{T}(f) \odot \mathbf{G}_{\mathrm{NLoS}}(f)\right)$ describes the contributions of NLoS paths in the channels from the BS to the $K$ UEs.

The effect of wave propagation along an edge in the graph as shown in Figure 1 can be calculated to be a multiplicative coefficient:

$$
A_{e}(f)=g_{e}(f) \exp \left(-j 2 \pi \tau_{e} f+j \phi\right),
$$

where $\tau_{e}$ denotes the time duration of the propagation along the edge, $\phi$ is a random initial phase which follows a uniform distribution on the interval $[0,2 \pi)$, and $g_{e}(f)$ represents the propagation coefficient which can be calculated based on the free-space propagation loss, reflection coefficient, or diffraction coefficient, depending on whether the edge is involved in $\mathbf{D}(f), \mathbf{R}(f), \mathbf{B}(f)$, or $\mathbf{T}(f)$. For the detailed information on how $g_{e}(f)$ is calculated, the readers are referred to [35].

The advantage of the graph model-based simulation lies in the low-complexity, as the CIR can be calculated analytically, and its capability of reproducing the specular-to-diffuse transition of the CIRs [34]. Furthermore, an environment can be easily modeled by the location matrices formed by involving the information of visibility and the locations of the BS, scatterers, and UEs. It has been shown in $[34,36,37]$ that, by appropriately tuning the model parameters, such as the exponent-decay constant of the path loss and including deterministic methods of computing diffraction coefficients, the CIRs obtained by graph modeling exhibit power delay profiles consistent with the real ones compared to the conventional ray-tracing-based method with tractable complexity. Furthermore, the applicability of the stochastic graph modelling has been widely validated in a variety of works, for example, the simulations of static in-room channel [35], body area channels [38], high-speed-railway channels [39], and multilink channels in indoor distributed antenna systems [36], as well as the analysis of MIMO channel's rank properties and capacity [40].

4.2. Simulated Environments and Specifications. In the simulations, an urban environment is considered where multiple scatterers are distributed as depicted in Figure 2. The green solid diamond in the figure represents the location of the BS

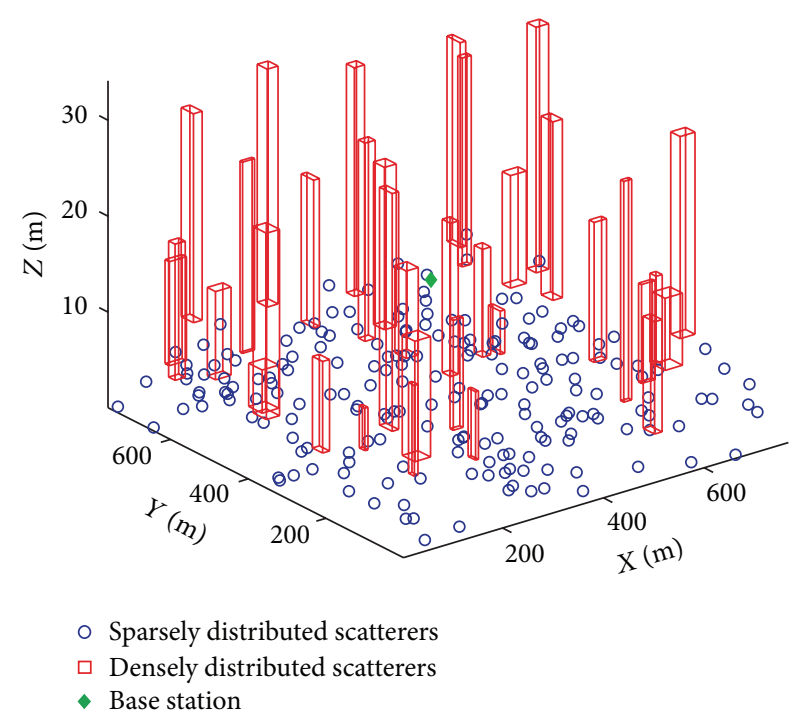

FIGURE 2: Propagation graph representation of the BS and densely and sparsely distributed scatterers for simulations.

TABle 1: Parameter settings for simulation.

\begin{tabular}{lc}
\hline Parameter & Setting \\
\hline Transmission bandwidth & $20 \mathrm{MHz}$ \\
Carrier frequency & $2.6 \mathrm{GHz}$ \\
Total UE locations & 2000 \\
BS antenna height & $20 \mathrm{~m}$ \\
Height of scatterers & $1-35 \mathrm{~m}$ \\
Height of UEs & $1-35 \mathrm{~m}$ \\
Signal to noise ratio & $30 \mathrm{~dB}$ \\
Half-power beamwidth in azimuth & $53^{\circ}$ \\
Half-power beamwidth in elevation & $79^{\circ}$ \\
Reflection loss & 0.7 \\
\hline
\end{tabular}

antenna with height of $20 \mathrm{~m}$. The red blocks denote the scope of the locations of scatterers densely distributed on the facades of buildings with significant physical extents. The buildings' heights range from 0 to $35 \mathrm{~m}$. The blue circles represent the slightly distributed scatterers corresponding to trees and bushes in the environment. The UEs are located randomly in the environment and their locations are assumed to be known. Table 1 reports the relevant parameter settings adopted in the simulations for generating the propagation graphs. Notice that since the BS antenna is surrounded by scatterers within a wide range of heights, some UEs are in LoS conditions with respect to the BS antenna and the others are in the NLoS conditions.

A directional antenna is considered in the BS side in the simulations in order to imitate the effect of sectoring coverage with different antenna attitude. Figure 3 illustrates the horizontal and vertical radiation pattern of the BS antenna. It can be observed from Figure 3 that the half-power beamwidth of the antenna is $79^{\circ}$ in azimuth and $53^{\circ}$ in elevation, which are close to the parameters of realistic BS antennas used in practice. In the simulations, propagation channels between 


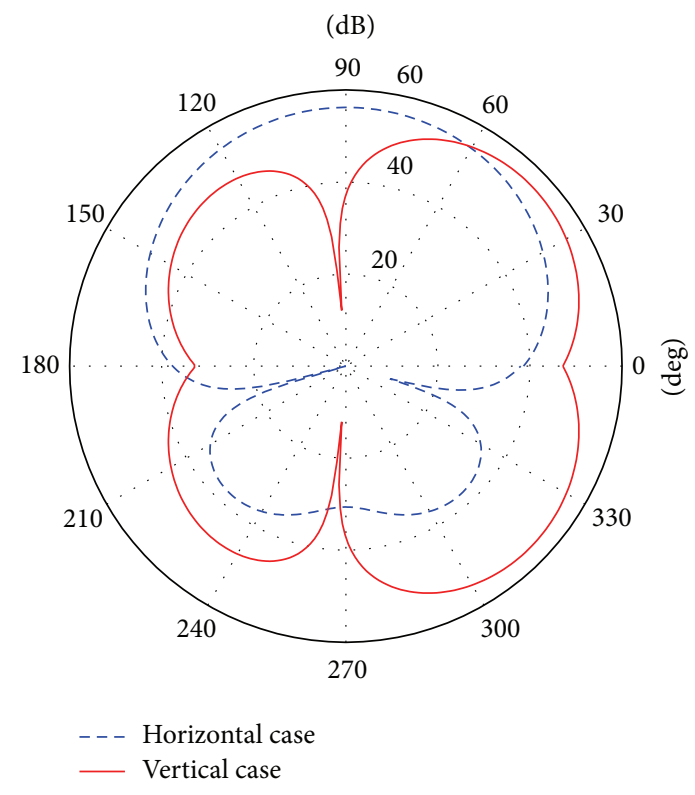

FIGURE 3: Normalized horizontal and vertical radiation patterns of the BS antenna adopted in the simulation.

the BS and the UEs are generated with the main beam of the BS antenna directed towards specific downtilt $\theta_{\mathrm{Tx}}$ and azimuth angle $\varphi_{\mathrm{Tx}}$. Considering that, in most cases the downtilt $\theta_{\mathrm{Tx}}$ is of more interest to be estimated than the azimuth angle $\varphi_{\mathrm{Tx}}$, we fix $\varphi_{\mathrm{Tx}}=0^{\circ}$ and adapt $\theta_{\mathrm{Tx}}$ within the range of $\left[0^{\circ}, 8^{\circ}\right]$ below the horizontal plane in the simulations.

4.3. Simulation Results and Analyses. To implement the CBE technique, the calibration is necessary to obtain path loss models and the statistics of shadow fading and multipath fading. In the simulations for the calibration, CIRs are generated for UEs in both LoS and NLoS scenarios by using a BS antenna with omnidirectional radiation pattern. Figures 4(a) and 4(b) show the examples of channel power delay profile (PDPs) obtained in, respectively, LoS and NLoS scenarios, where the direct distance between the BS and UE equals $350 \mathrm{~m}$ and the signal to noise power ratio (SNR) is set to $30 \mathrm{~dB}$. It is evident from Figure $4(\mathrm{a})$ that the power of the LoS component is dominant in the PDP in the LoS scenario and, for NLoS case shown in Figure 4(b), multipath propagation effect is more serious as the LoS path no longer exists.

Figures 5(a) and 5(b) illustrate the scatter plots of path loss versus the BS-UE separation $d$ for the LoS and NLoS scenarios, respectively. Regression lines expressed as $g_{p}(d)=$ $20 \times \log _{10} d+44.5$ and $g_{p}(d)=23 \times \log _{10} d+64.4$ are adopted to fit the empirical data for LoS and NLoS cases, respectively. It can be observed from Figures 5(a) and 5(b) that the path loss varies differently versus $d$ for LoS and NLoS scenarios. This reveals the necessity of modeling the fading statistics for the LoS and NLoS channels separately. Furthermore, it is worth to mention that the parameters of the fitted lines in Figures 5(a) and 5(b) have values similar to those specified for the LoS and NLoS path loss models

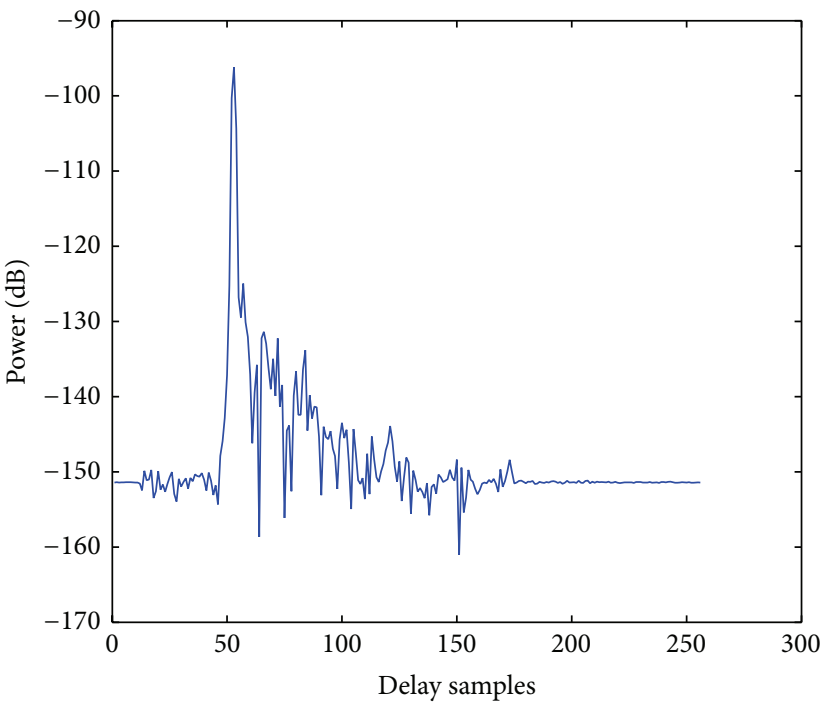

(a) An example of PDP in a LoS scenario

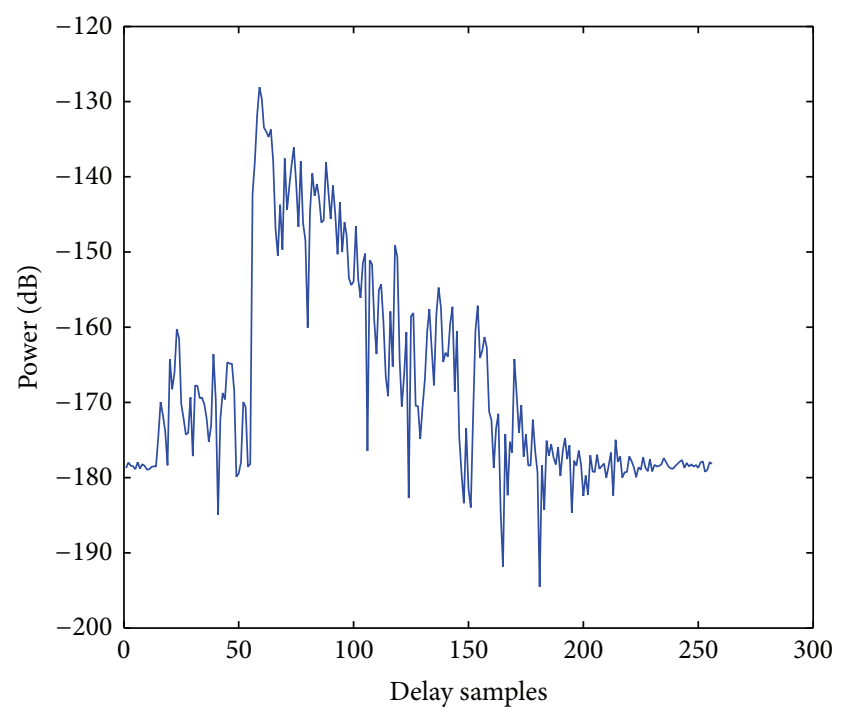

(b) An example of PDP in a NLoS scenario

FIGURE 4: Simulation comparison of PDPs for LoS and NLoS scenarios with similar distances.

in the WINNER II standards [41]. Figures 6(a) and 6(b) depict, respectively, the empirical pdfs of the shadow fadings expressed in $\mathrm{dB}$ and of multipath fadings in the linear scale without distinguishing whether UEs are in LoS or NLoS scenarios. It can be observed that the empirical distributions of the shadowing and multipath fading can be well fitted with the normal and Rician distribution, respectively. The parameters of the fitted pdfs are reported in the legends of Figures 6(a) and 6(b).

In the simulations, the effect of the directive radiation pattern with specific downtilt is considered when generating individual propagation paths between scatterers and the BS antenna. Both the CBE and the CFE methods are applied to estimate $\theta_{\text {Tx }}$ based on the received signal strength reported by UEs in either LoS or NLoS conditions. Figure 7 demonstrates 


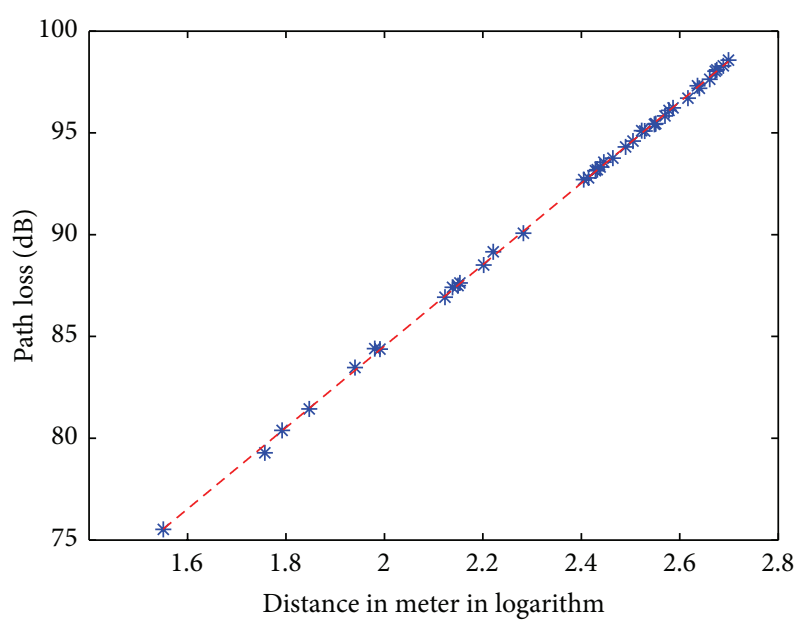

* Simulation results - - - Slope $n=2.00$

(a) Path loss in LoS scenarios

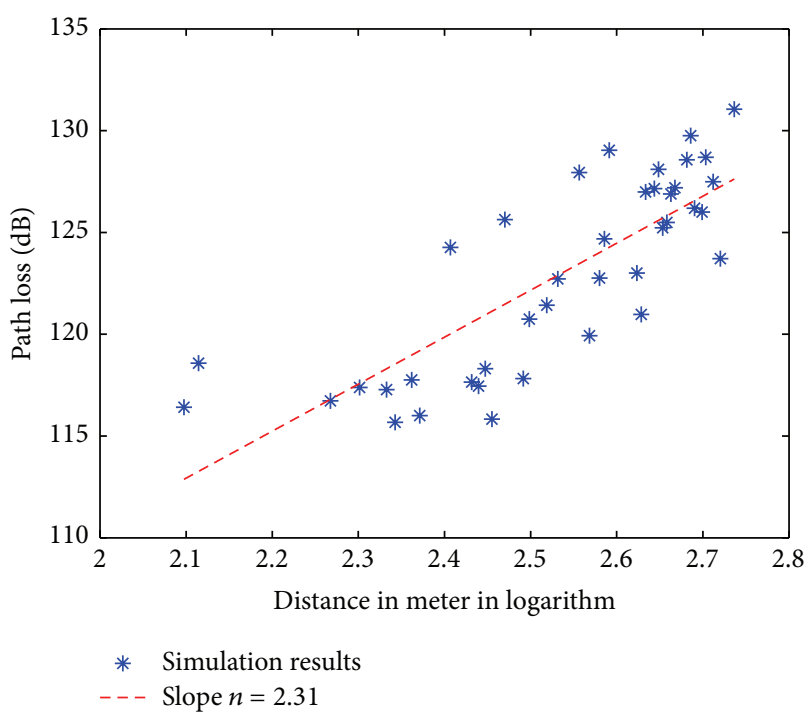

(b) Path loss in NLoS scenarios

FIGURE 5: Path loss models extracted for the simulation results obtained in LoS and NLoS scenarios.

the objective function $\Lambda\left(\theta_{\mathrm{TX}} ; \mathbf{g}, \sigma_{s}, \mu_{m}, \sigma_{m}\right)$ calculated in one simulation snapshot where the true downtilt $\theta_{\mathrm{Tx}}^{\prime}$ is set to $4^{\circ}$. It can be observed from Figure 7 that the maximum of the objective function is achieved at $\theta_{\mathrm{Tx}}=4^{\circ}$. Thus, the downtilt of the BS antenna is correctly estimated in this snapshot.

To illustrate the performance of the $\mathrm{CBE}$ and CFE techniques more visually, Figure 8 compares the true BS antenna radiation pattern $g_{\mathrm{Tx}}\left(\boldsymbol{\Omega}_{\mathrm{Tx}}\right)$ with $\theta_{\mathrm{Tx}}^{\prime}=4^{\circ}$ observed by UEs under the assumption that they are all in the LoS conditions, with the estimate $\widehat{g}_{\mathrm{Tx}}\left(\boldsymbol{\Omega}_{\mathrm{Tx}}\right)$ calculated with candidate downtilt $\widehat{\theta}_{\mathrm{TX}}=4^{\circ}$ based on the received signal strengths reported by UEs in either LoS or NLoS scenarios. It can be observed that the true and the estimated BS radiation patterns look similar to each other. However, by careful examination, we found that the estimated radiation pattern

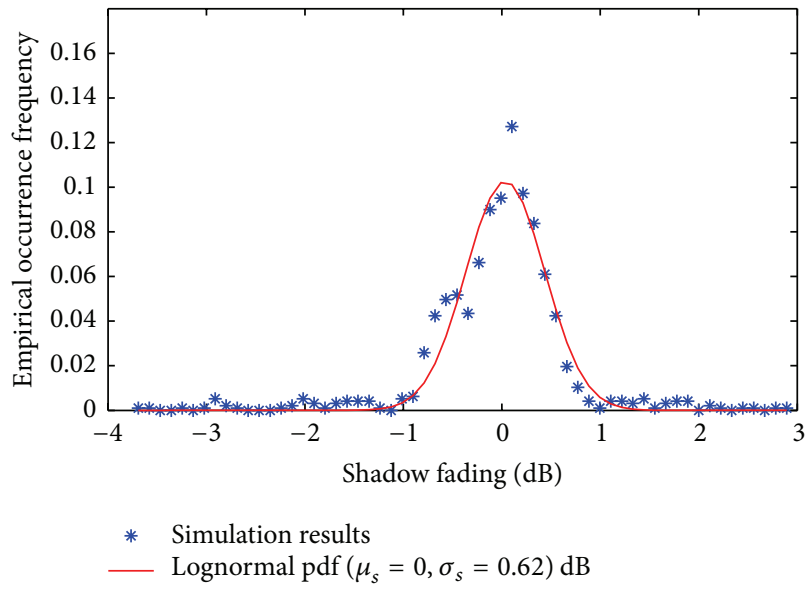

(a) Shadow fading

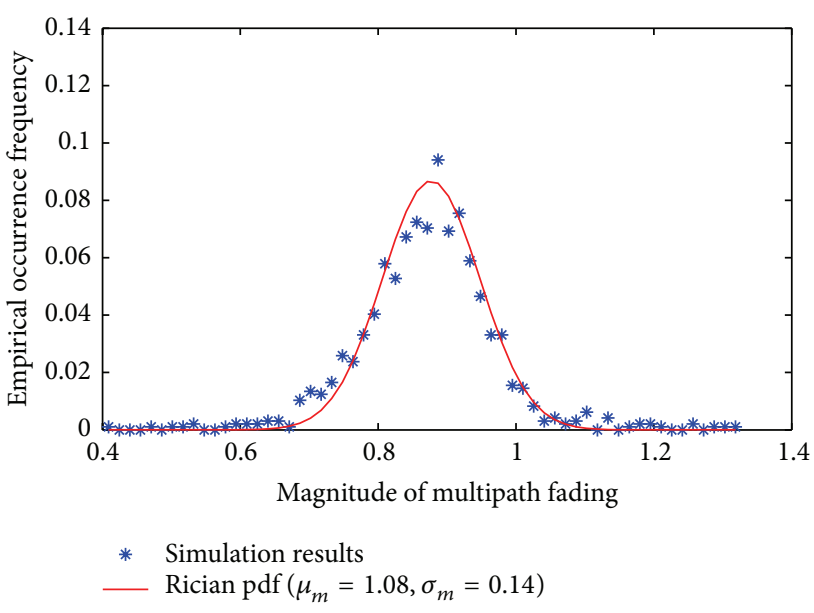

(b) Multipath fading

FIgURE 6: Scatter plots of the empirical pdfs of shadow fading and multipath fading observed by all UEs regardless of LoS and NLoS scenarios.

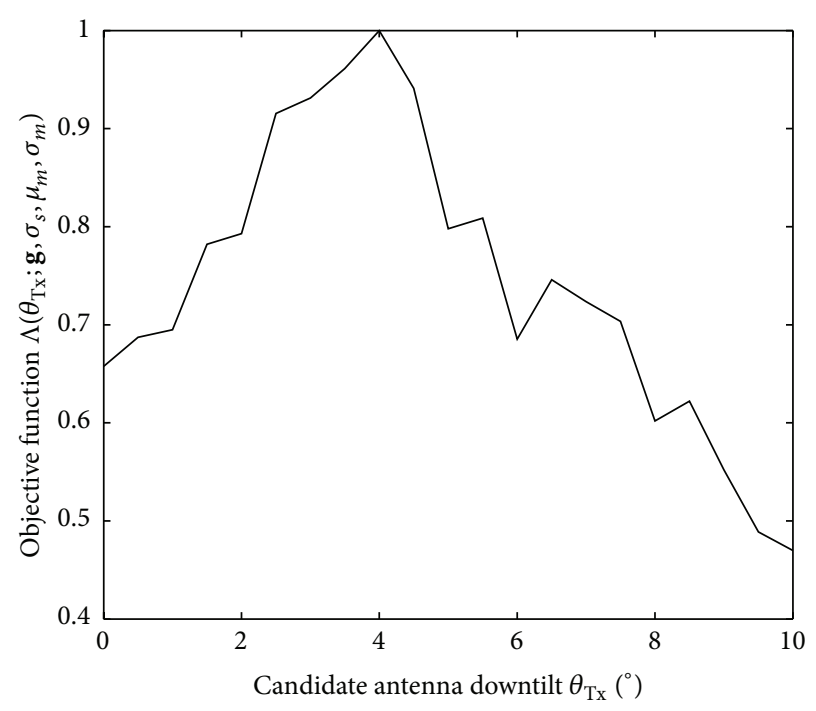

FIGURE 7: An example of the objective function of downtilt calculated in a simulation with the true downtilt $\theta_{\mathrm{Tx}}^{\prime}=4^{\circ}$. 


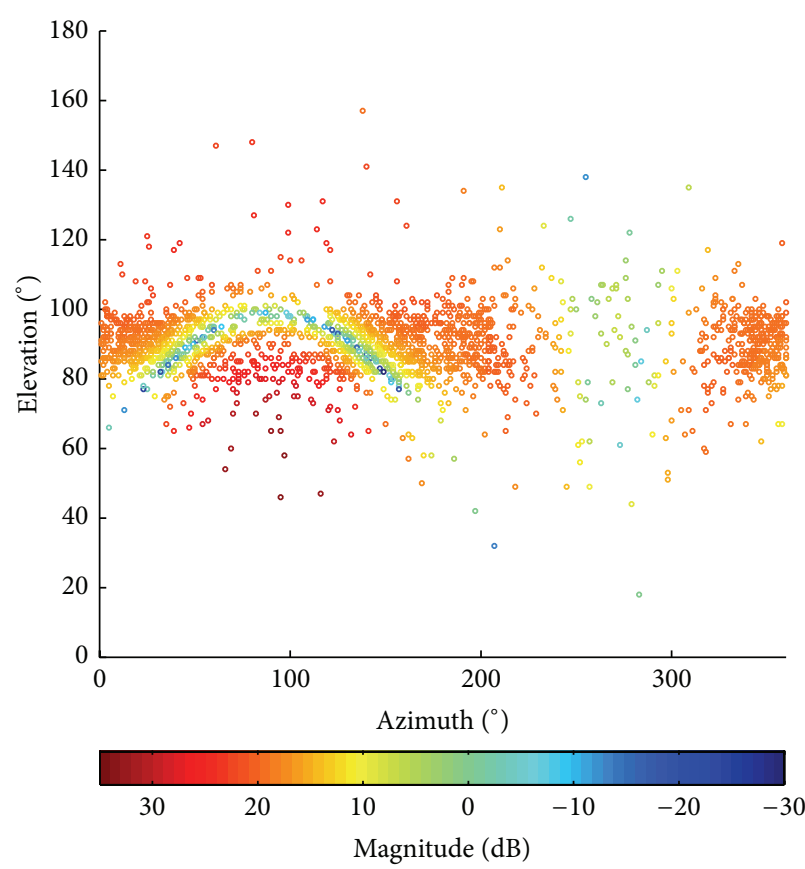

(a) Original BS radiation pattern

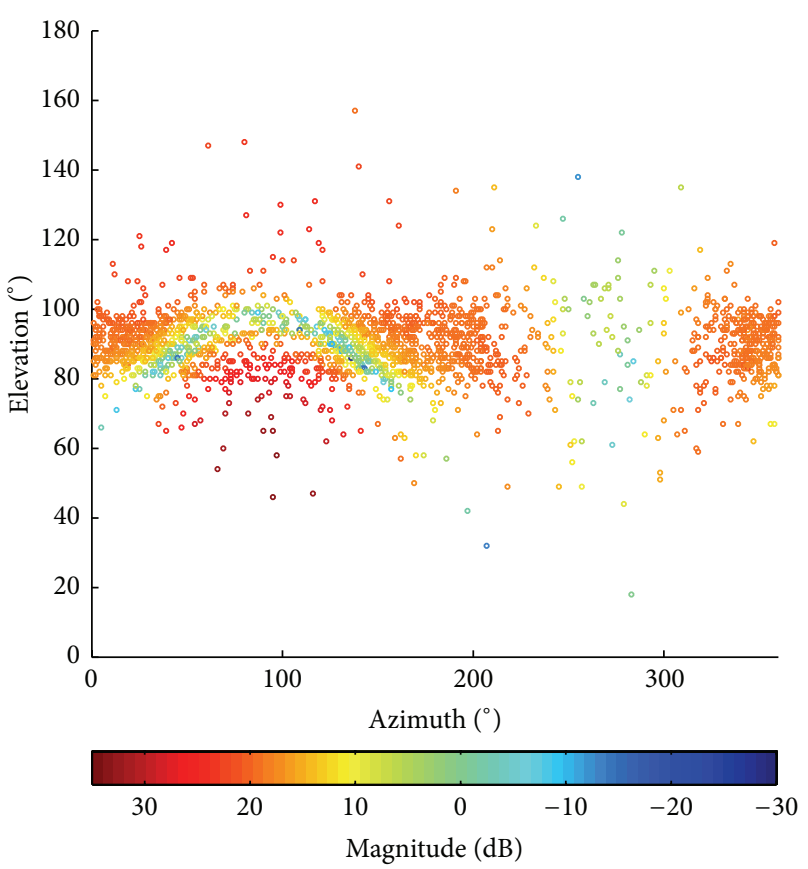

(b) Estimated BS radiation pattern

FIGURE 8: The original and estimated BS antenna radiation patterns.

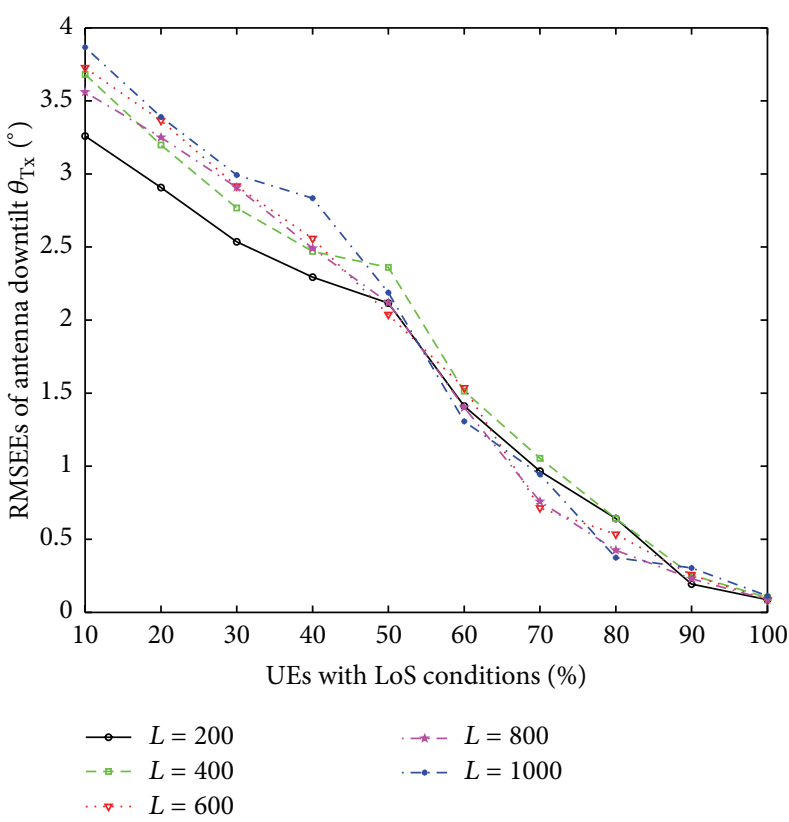

(a) The CBE method

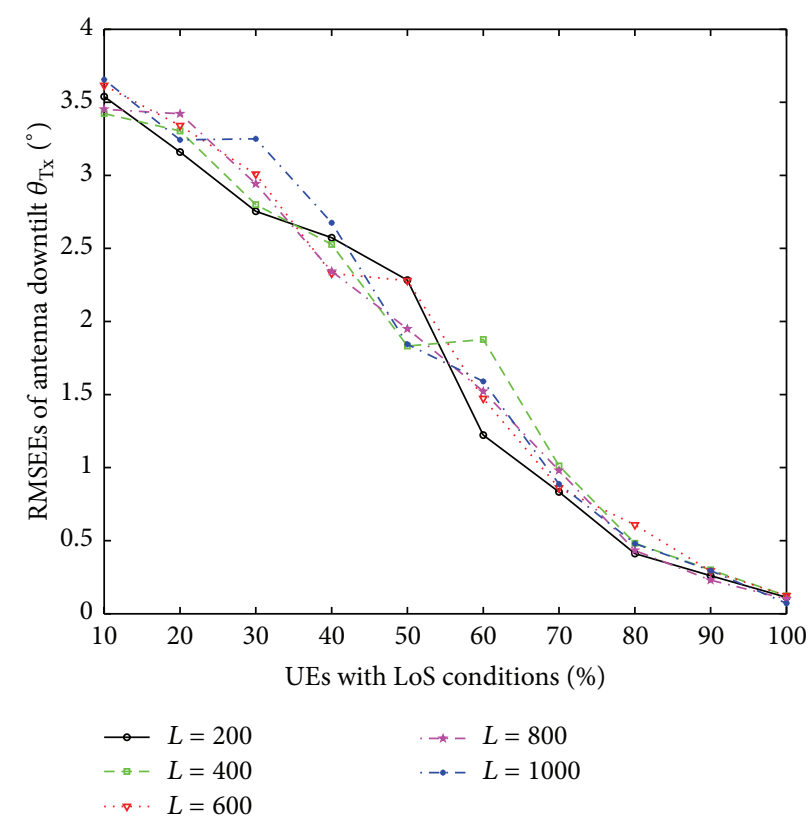

(b) The CFE method

FIGURE 9: The RMSEEs of antenna downtilt versus the percentage of the LoS users with the total number of users as a parameter.

appears more blurred than the true counterpart at the lowgain portions of both patterns which are colored in green or blue in Figures 8(a) and 8(b). This may be due to two reasons: first, the random variation of the shadow fading and multipath fading can lead to the uncertainty in $\widehat{g}_{\mathrm{Tx}}\left(\boldsymbol{\Omega}_{\mathrm{Tx}}\right)$; second, the assumption adopted in the estimation that the DoDs of all paths between the BS and a UE are identical to the DoD of the LoS path is inaccurate and introduces some errors in calculating $\widehat{g}_{\mathrm{Tx}}\left(\boldsymbol{\Omega}_{\mathrm{Tx}}\right)$.

Figures 9(a) and 9(b) illustrate, respectively, the root mean square estimate errors (RMSEEs) of the antenna downtilt $\theta_{\mathrm{Tx}}$, denoted by $\operatorname{RMSEE}\left(\theta_{\mathrm{Tx}}\right)$ obtained by using the CBE and CFE methods versus the percentage of LoS UEs among all UEs with true $\theta_{\mathrm{Tx}}=4^{\circ}$. The total number $L$ of the UEs is 

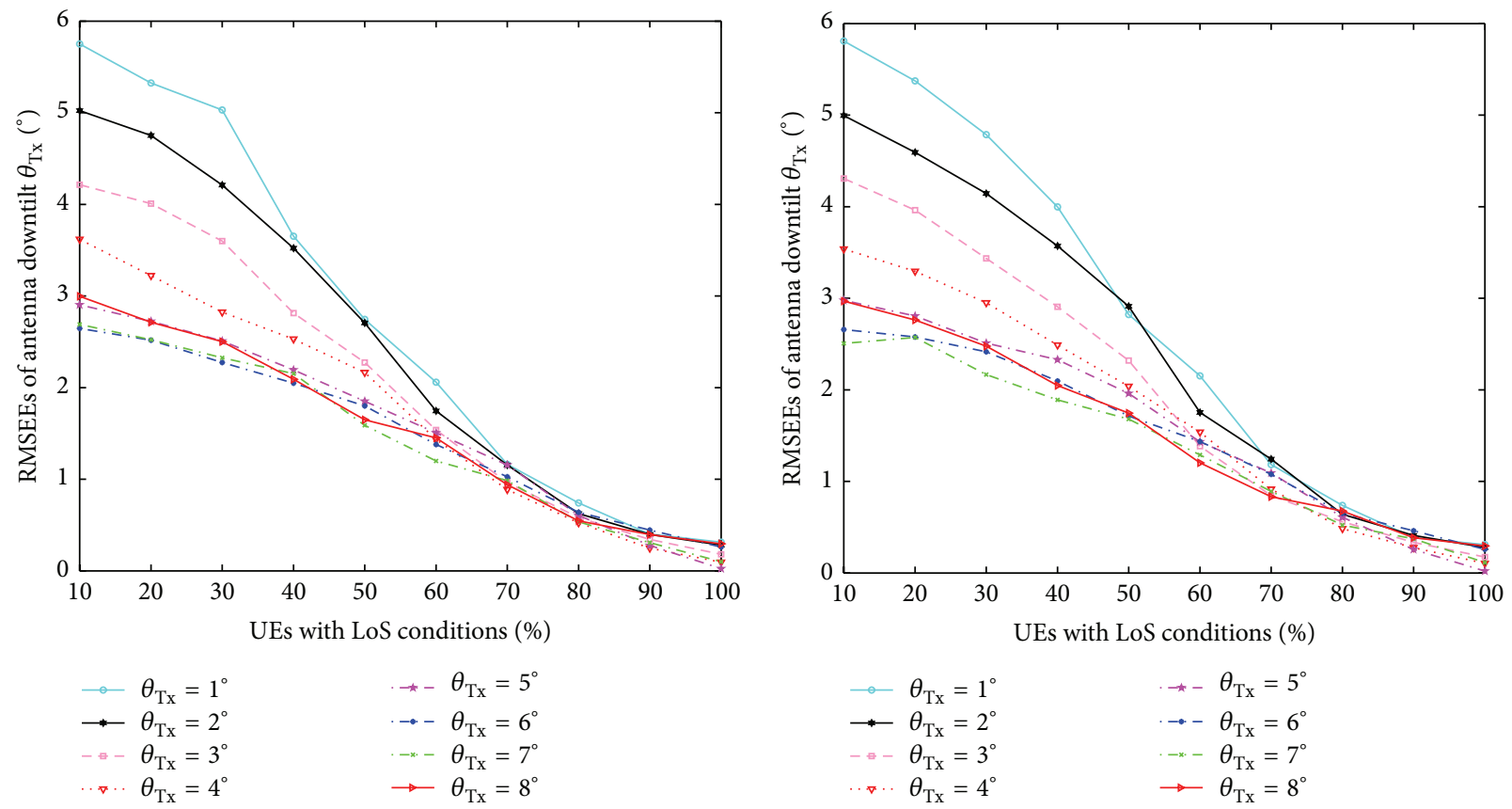

(a) The CBE method

(b) The CFE method

FIgURE 10: The RMSEEs of the antenna downtilt versus the percentage of the UEs in the LoS positions among all available UEs.

considered as a parameter ranging from 200 to 1000. It can be observed from Figures 9(a) and 9(b) that, for both methods, the RMSEE $\left(\theta_{\mathrm{Tx}}\right)$ decreases linearly with respect to the LoS percentage. Specifically, when the LoS percentage is beyond $70 \%$, the $\operatorname{RMSEE}\left(\theta_{\mathrm{Tx}}\right)$ decreases to zero regardless of $L$. The value of $L$ exhibits insignificant impacts on the behavior of $\operatorname{RMSEE}\left(\theta_{\mathrm{Tx}}\right)$. Additionally, we observe from Figure 9(a) that, for the CBE method, when the percentage of LoS users is less than $50 \%$, the $\operatorname{RMSEE}\left(\theta_{\mathrm{Tx}}\right)$ increases along with $L$. In the cases with the LoS percentage equal to $10 \%$, the performance obtained with the least number of users considered, that is, $L=200$, turns out be the best among all choices of $L$. This phenomenon is unobserved when the CFE method is applied, as illustrated in Figure 9(b) where the $\operatorname{RMSEE}\left(\theta_{\mathrm{Tx}}\right)$ graphs versus the LoS percentages are indistinguishable for various $L$. The effect that the CBE is more sensitive to the value of $L$ than the CFE may be due to the reason that the calibrated path loss models and statistical parameters are more accurate in the cases where more UEs are considered. When $L$ reduces, the deviations between the true model, including the parameters, and the calibration results increases and leads to larger RMSEE $\left(\theta_{\mathrm{Tx}}\right)$ consequently.

Figures 10(a) and 10(b) depict, respectively, $\operatorname{RMSEE}\left(\theta_{\mathrm{TX}}\right)$ obtained by using the CBE and CFE methods versus the percentage of the UEs in the LoS positions among all considered UEs. The true $\theta_{\mathrm{Tx}}$ is a parameter ranging from $1^{\circ}$ to $8^{\circ}$. It can be observed from Figures $10(\mathrm{a})$ and $10(\mathrm{~b})$ that the $\operatorname{RMSEE}\left(\theta_{\mathrm{TX}}\right)$ obtained with the CBE and CFE decreases with respect to the LoS percentage for all values of $\theta_{\mathrm{Tx}}$ considered. Furthermore, the value of the true $\theta_{\mathrm{Tx}}$ also significantly influences the performance of both methods. To be specific, less $\operatorname{RMSEE}\left(\theta_{\mathrm{TX}}\right)$ is obtained for larger $\theta_{\mathrm{TX}}$. This is reasonable

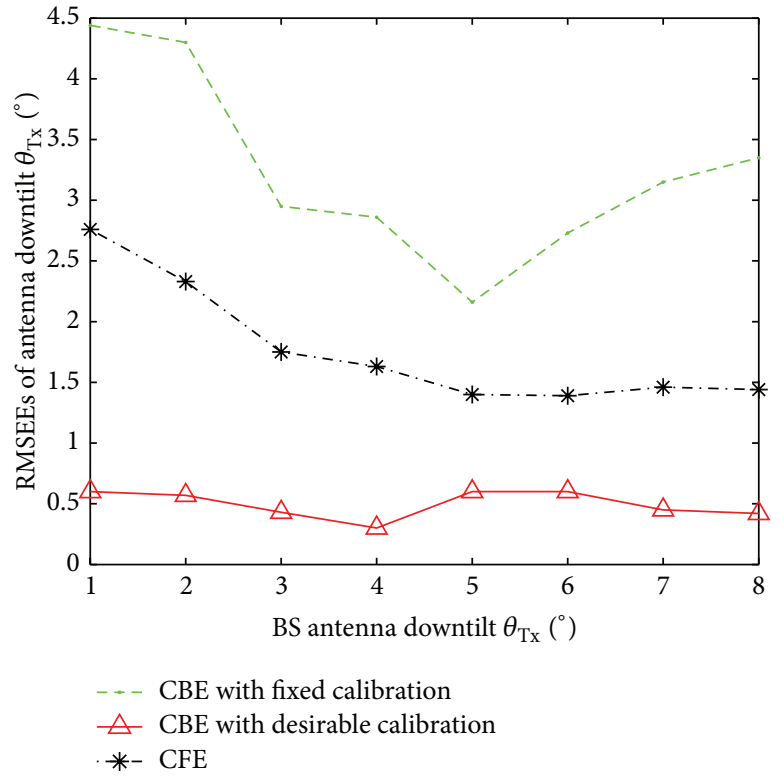

FIgURE 11: $\operatorname{RMSEE}\left(\theta_{\mathrm{Tx}}\right)$ versus $\theta_{\mathrm{Tx}}$ obtained by using the CFE method and two CBE methods which make use of, respectively, "desirable" or "fixed" calibration results.

since the number of the UEs that are distributed in the main beam of the antenna increases along with $\theta_{\mathrm{Tx}}$. In addition, it is evident by comparing Figures 10(a) and 10(b) that the CBE and CFE methods exhibit quite similar $\operatorname{RMSEE}\left(\theta_{\mathrm{Tx}}\right)$ graphs. We postulate that this is because, in most cases, the assumed types of distributions for shadowing and multipath fading 


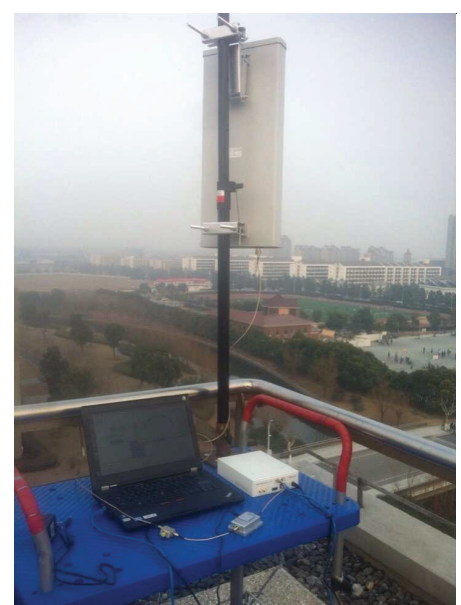

(a) $\mathrm{Tx}$

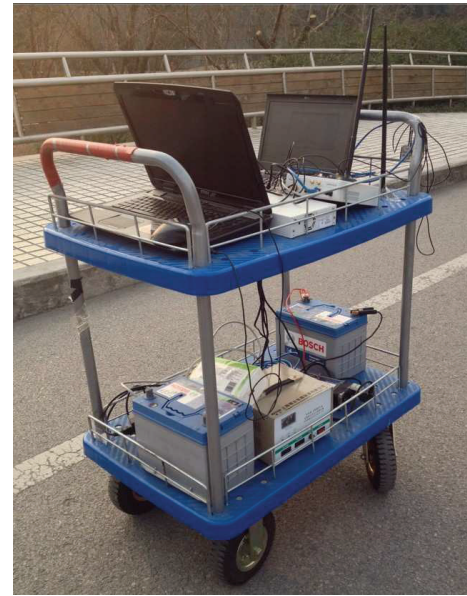

(b) $\mathrm{Rx}$

Figure 12: The photographs of the Tx and Rx in the measurement campaign.

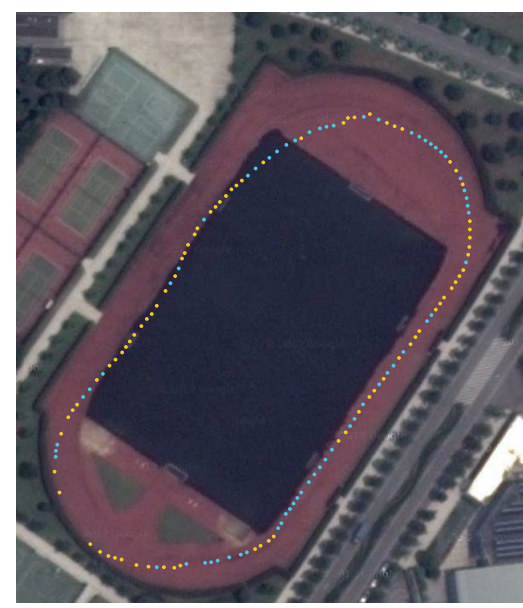

(a) LoS/NLoS positions visually identified

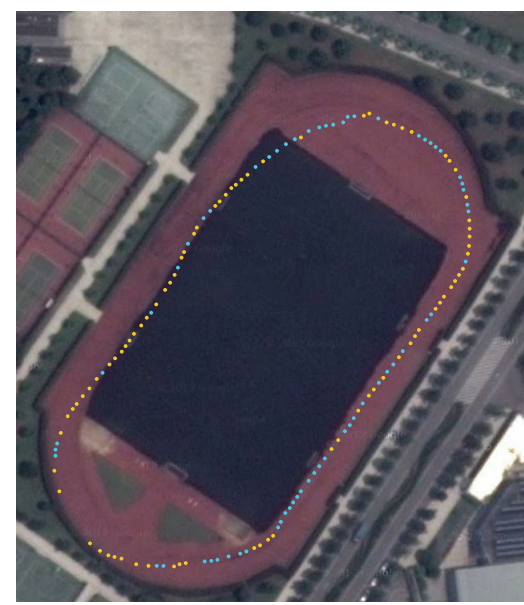

(b) LoS/NLoS positions identified by checking delay spread

FIGURE 13: Comparison of the LoS/NLoS positions visually identified and detected based on delay spread.

that the CFE method relies on contain the equivalent information as those carried by the calibration results adopted in the CBE method. As a result, the CBE method does not yield improved performance, compared with the CFE method.

Figure 11 compares the graphs of $\operatorname{RMSEE}\left(\theta_{\mathrm{Tx}}\right)$ versus $\theta_{\mathrm{Tx}}$ obtained by using the CFE method and two CBE methods which make use of, respectively, "desirable" or "fixed" calibration results. Here, "desirable" refers to the situation where the $\mathrm{CBE}$ is performed by using the path loss model and fading statistics calibrated with BS antenna downtilt set to be identical to the unknown $\theta_{\mathrm{Tx}}$, and "fixed" denotes the case where the calibration results are obtained with a fixed BS antenna downtilt which is unnecessary to be identical to the exact $\theta_{\mathrm{Tx}}$. It can be observed from Figure 11 that if the calibration campaign is not carried out with BS antenna downtilt equal to the exact $\theta_{\mathrm{Tx}}$ to estimate, the CFE method is superior to the $\mathrm{CBE}$ in terms of lower $\operatorname{RMSEE}\left(\theta_{\mathrm{Tx}}\right)$. Notice that, in real cases where $\theta_{\mathrm{Tx}}$ is unknown, it is impossible to determine which calibration result is "desirable." Thus, in such cases the CFE method is more preferable than the CBE method for attitude estimation.

\section{Experimental Investigation}

5.1. Measurement Equipment and Campaign Specifications. To evaluate the performances of the CBE and CFE methods, experimental investigations have been conducted. Figures 12(a) and 12(b) illustrate, respectively, the photographs of the $\mathrm{Tx}$ and the Rx used in the measurement campaign. The Tx, located on the rooftop of a 7-storey building, was applied as a BS. The $\mathrm{Rx}$, loaded on a trolley which moved in the ground level, is considered as a UE. The Tx made use of a universal software-defined radio peripheral (USRP) of type N210 broadcasting repetitively a pseudonoise sequence with Gaussian-pulse-shaping and quadrature phase shift keying (QPSK) modulation through a directional planar antenna as 


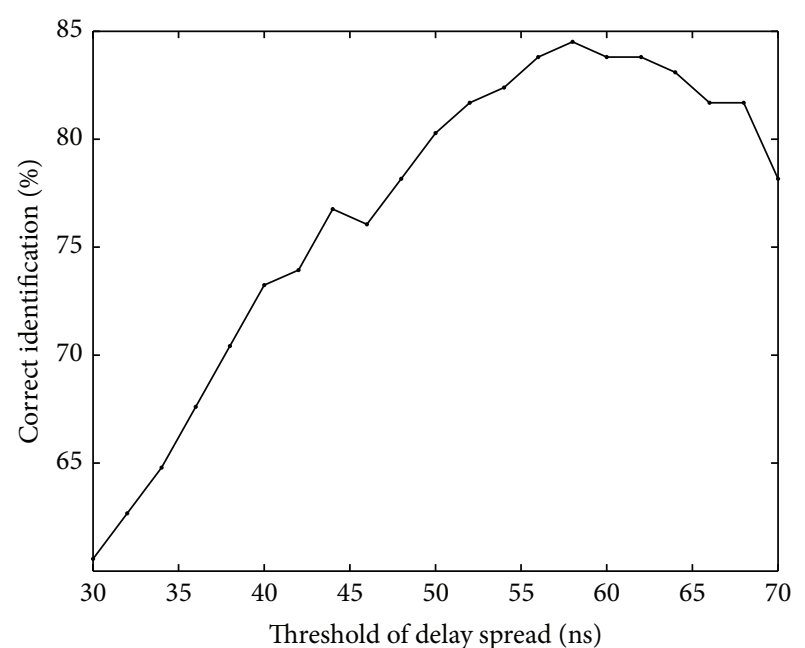

FIGURE 14: Percentage of correct identification versus the threshold of delay spread.

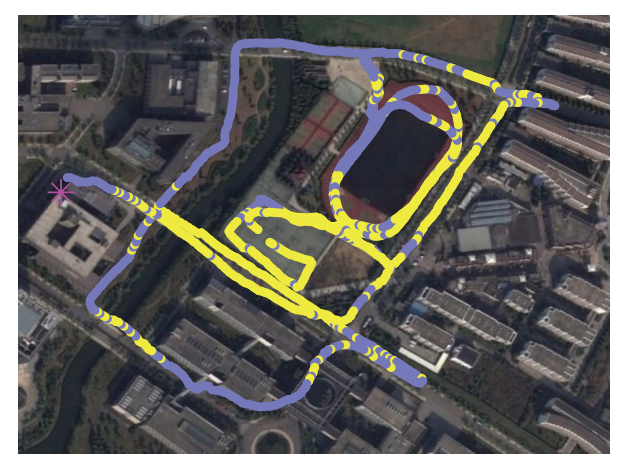

FIgURE 15: The map of the environment, the location of the Tx antenna, and the routes along which the $\mathrm{Rx}$ moves during the measurements.

shown in Figure 12(a). In the Rx side, another USRP received the signals through a dipole antenna under the control of the GNU radio software. A global positioning system (GPS) module was installed in the $\mathrm{Rx}$ to record the longitudes and latitudes of the measurement locations. Furthermore, a power amplifier with output signal power up to $37 \mathrm{dBm}$ was used in the Tx.

The measurement campaigns, including those applied for the calibration of the path loss models and fading statistics, have been conducted in the Jiading Campus of Tongji University, Shanghai, China, in January 2014. The campus is a typical urban scenario with densely distributed buildings and plants. Figure 15 illustrates the map of the part of the campus where the measurements were performed. The yellow asteroid shown in Figure 15 represents the Tx location and the green arrowhead points to the due North direction. During the measurements, the $\mathrm{Rx}$ loaded in a trolley moved at a walking speed of $3 \mathrm{~km} / \mathrm{h}$ along predefined routes as depicted in Figure 15. Once the $\mathrm{Rx}$ moved away from its previous location by $3 \mathrm{~m}$, data acquisition was activated and lasted for $1 \mathrm{~s}$. The signal bandwidth was $10 \mathrm{MHz}$. The carrier frequency
TABLE 2: Parameter settings used in the measurements.

\begin{tabular}{lc}
\hline Parameter & Setting \\
\hline Antenna frequency band & $1.710-2.5 \mathrm{GHz}$ \\
Antenna voltage standing wave ratio & $\leq 1.5$ \\
Effective antenna gain & $12.3 \mathrm{dBi}$ \\
Gain of power amplifier & $37 \mathrm{dBm}$ \\
BS height & $25 \mathrm{~m}$ \\
Center frequency & $2.4 \mathrm{GHz}$ \\
Transmitting bandwidth & $5 \mathrm{MHz}$ \\
Transmitting power & $20 \mathrm{~dB}$ \\
Receiving bandwidth & $10 \mathrm{MHz}$ \\
Moving speed of UE & $3 \mathrm{~km} / \mathrm{h}$ \\
Total UE number & 1100 \\
BS antenna downtilt $\theta_{\text {Tx }}$ & $3^{\circ}, 5^{\circ}, 6^{\circ}, 8^{\circ}, 10^{\circ}$ \\
BS antenna azimuth $\varphi_{\mathrm{Tx}}$ & $115^{\circ}$ \\
Horizontal half-power beamwidth & $56^{\circ}$ \\
Vertical half-power beamwidth & $14^{\circ}$ \\
Coverage distance $d_{\mathrm{C}}$ & $476 \mathrm{~m}$ \\
\hline
\end{tabular}

was $2.4 \mathrm{GHz}$. Table 2 reports the setting parameters of the measurements. In the measurement campaign, the Rx moved along the same routes for totally seven times. In five of them, the Tx antenna downtilt $\theta_{\mathrm{Tx}}$ was set to $3^{\circ}, 5^{\circ}, 6^{\circ}, 8^{\circ}$, and $10^{\circ}$, respectively, and in the other two the Tx antenna azimuth $\varphi_{\mathrm{Tx}}$ was set to $25^{\circ}$ and $0^{\circ}$, respectively. Here, a direction with $\varphi_{\mathrm{Tx}}=0^{\circ}$ coincides with the due North direction. The measurement data was applied to estimate the CIRs by correlating the received signals in baseband representation with the calibrated PN sequence which was obtained by directly connecting the USRPs in the Tx and the Rx.

5.2. Evaluation of LoS Detection Based on Delay Spread. The delay spread is defined as the second central order moment of the normalized PDP of a channel [41]. It has been found that the delay spread of NLoS channels can be several times larger than that for the LoS cases in a residential environment [33, 42]. Inspired by these results, we use the delay spread as an indicator to distinguish LoS and NLoS channels. In order to find out a suitable threshold of delay spread which is applicable to determine whether the LoS exists for the environment of interest, measurement campaign was carried out where the $\mathrm{Rx}$ is on the playground and the Tx is deployed on the top of one building. During the measurements, the locations have LoS connections to the Tx which were visually identified. Figure 13(a) illustrates the locations of the measurements on the playground, among which those with brown and blue spots indicate, respectively, the visually identified LoS and NLoS positions. In order to find the most appropriate value for the threshold delay in LoS/NLoS detection, the correct identification percentage is investigated with respect to different candidates of threshold delay spread. Figure 14 depicts the graph obtained, where the correct detection percentage achieves the maximum of $85 \%$ when the threshold delay spread is chosen to be 55 ns. Figure 13(b) shows the LoS/NLoS detection results obtained by applying $55 \mathrm{~ns}$ as 
a threshold value for delay spread. By comparing Figures 13(a) and 13(b), it is obvious that, in most locations, the LoS situation is correctly identified. Thus, practically, we use 55 ns as the delay spread threshold for LoS/NLoS detection based on the CIRs. Figure 15 depicts all measurement locations of the measurements where the LoS exists only in the locations marked by yellow spots. The pink star shows the location of Tx.

\subsection{Measurement Results}

5.3.1. Performance of the CBE Method. The measurement data collected with different settings of $\theta_{\mathrm{Tx}}$ is used to calibrate $\boldsymbol{\kappa}$ for specific downtilts, which are applied in the CBE method to estimate $\theta_{\mathrm{Tx}}$. Results show that the CBE method provides the lowest $\operatorname{RMESS}\left(\theta_{\mathrm{Tx}}\right)$ with $\boldsymbol{\kappa}$ obtained with $\theta_{\mathrm{Tx}}=3^{\circ}$ and returns the largest $\operatorname{RMESS}\left(\theta_{\mathrm{Tx}}\right)$ with $\boldsymbol{\kappa}$ obtained with $\theta_{\mathrm{Tx}}=10^{\circ}$. This performance degradation is probably due to the less accuracy of $\boldsymbol{\kappa}$ when the calibration is performed with large antenna downtilt; for example, $\theta_{\mathrm{Tx}}=10^{\circ}$. When $\theta_{\mathrm{Tx}}$ increases up to a certain level, the region covered by the antenna main beam shrinks and, as a result, the number of UEs with strong received signals decreases, leading to a lower SNR which deteriorates the calibration accuracy. To avoid selecting the calibrated $\boldsymbol{\kappa}$ with a specific $\theta_{\mathrm{Tx}}$, we propose a modified CBE which finds $\widehat{\theta}_{\mathrm{Tx}}$ by maximizing the aggregated likelihood function of $\theta_{\mathrm{Tx}}$ calculated based on all available calibrated $\boldsymbol{\kappa}$. Figure 16 compares the $\operatorname{RMSEE}\left(\theta_{\mathrm{Tx}}\right)$ obtained by using the modified CBE method and two original CBE methods which utilize $\boldsymbol{\kappa}$ obtained with $\theta_{\mathrm{Tx}}=3^{\circ}$ and $10^{\circ}$, respectively. It can be observed from Figure 16 that the performance of the modified CBE method is close to the best performance which can be achievable by using the original CBE method.

5.3.2. Performance Evaluation for the CFE Method. The performance of the CFE method is also evaluated based on the measurement data collected with different settings of $\theta_{\mathrm{Tx}}$. To investigate the stochastic behavior of $\operatorname{RMSEE}\left(\theta_{\mathrm{Tx}}\right)$, the $\mathrm{CFE}$ is applied in multiple snapshots. In each snapshot, a certain number of measurement locations is randomly selected from all locations, and the received signal strengths obtained from these locations, including LoS and NLoS scenarios, are applied to estimate $\theta_{\mathrm{Tx}}$. Figure 17 illustrates the $\operatorname{RMSEE}\left(\theta_{\mathrm{Tx}}\right)$ versus the percentage of the LoS locations out of all selected locations, with the value of the true $\theta_{\mathrm{Tx}}$ ranging from $3^{\circ}$ to $10^{\circ}$. It can be observed from Figure 17 that the $\operatorname{RMSEE}\left(\theta_{\mathrm{Tx}}\right)$ decreases when the percentage of the LoS locations increases. This is consistent with the simulation results illustrated in Section 4.3. Furthermore, it is also observed that, for a fixed percentage, the $\operatorname{RMSEE}\left(\theta_{\mathrm{Tx}}\right)$ increases when the true $\theta_{\mathrm{Tx}}$ increases. We postulate that this is due to the fact that, with a larger $\theta_{\mathrm{Tx}}$, the BS antenna radiation pattern is more subject to the distortions caused by the premises around the BS in such a way that the true antenna radiation pattern is significantly different from that adopted in the CFE method.

It is worth mentioning that the observation of larger $\operatorname{RMSEE}\left(\theta_{\mathrm{Tx}}\right)$ when $\theta_{\mathrm{Tx}}$ increases in the experimental investigation is contradictory to the simulation results shown in

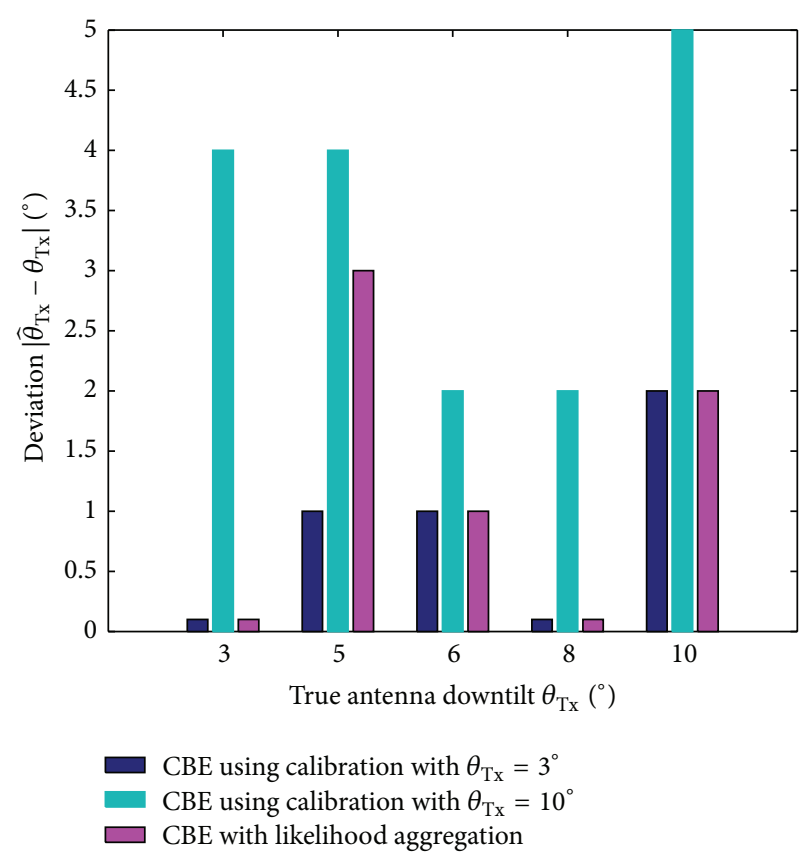

FIGURE 16: Deviation $\left|\widehat{\theta}_{\mathrm{Tx}}-\theta_{\mathrm{Tx}}\right|$ between the true antenna downtilt and the estimates obtained by using the CBE method.

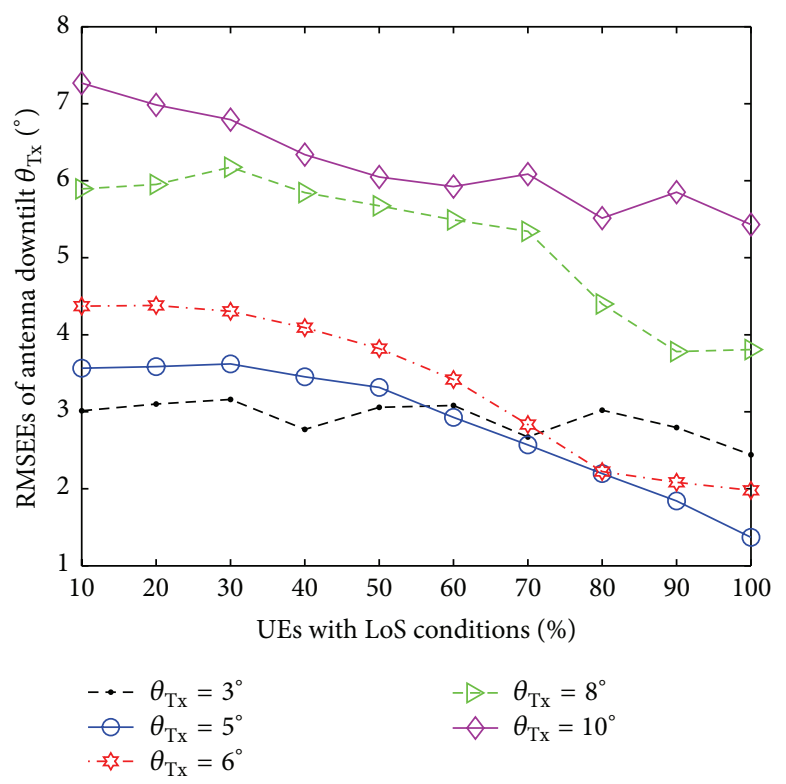

FIGURE 17: The RMSEEs of antenna downtilt $\theta_{\text {Tx }}$ obtained by using the CFE method versus the percentage of the UEs in the LoS conditions among all UEs.

Figure 10(b) where lower $\operatorname{RMSEE}\left(\theta_{\mathrm{Tx}}\right)$ is obtained when $\theta_{\mathrm{Tx}}$ increases. Our conjecture of the reason of such a difference is that, in the experiments, the performance degradation due to the distorted antenna radiation pattern, which was not considered in the simulation, may be more severe than the performance improvement which benefited from more users being involved when $\theta_{\mathrm{Tx}}$ increases and, consequently, the $\operatorname{RMSEE}\left(\theta_{\mathrm{Tx}}\right)$ deteriorates. 


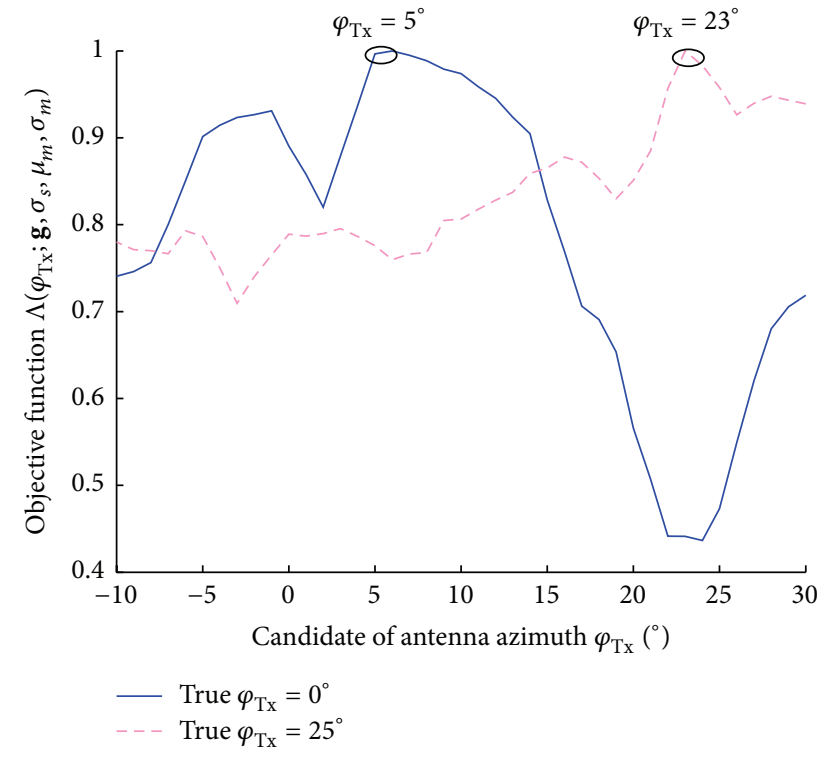

FIGURE 18: The objective functions calculated in the CFE method with true $\varphi_{\mathrm{Tx}}=0^{\circ}$ and $25^{\circ}$, respectively.

To our knowledge, most conventional studies focus on the influence of the antenna downtilt and provide only empirical equations of the optimal downtilt angle of the antenna, rather than an estimation procedure as what we proposed in the our work. Furthermore, rare literature is related to estimating the BS antenna attitude, except for optimal antenna attitude (downtilt) calculation based on the following equations [19, 20]:

$$
\begin{aligned}
& \theta_{\mathrm{opt}}=\arctan \left(\frac{h_{\mathrm{BS}}-h_{\mathrm{MS}}}{d_{\mathrm{C}}}\right)+\frac{\theta_{-3 \mathrm{~dB}}}{2}, \\
& \theta_{\mathrm{opt}}=3\left[\ln \left(h_{\mathrm{BS}}\right)-d_{\mathrm{C}}^{0.8}\right] \log _{10}\left(\theta_{-3 \mathrm{~dB}}\right),
\end{aligned}
$$

where $h_{\mathrm{BS}}$ and $h_{\mathrm{MS}}$ denote the height of the BS and UEs, respectively, $d_{C}$ represents the coverage distance, and $\theta_{-3 \mathrm{~dB}}$ means the vertical half-power beamwidth. Equation (12) leads to significant overestimation or underestimation with regard to the optimal downtilt angle. For the method in (13), the parameters adopted are quite site-specific, which reduce its applicability in general cases significantly. In the measurement, using the statistics in Table 2, the optimal antenna downtilt can be calculated by using (12) and (13) to be $9.86^{\circ}$ and $9.17^{\circ}$, respectively, for different practical antenna downtilts. These results yield larger estimated errors than our proposed method obviously.

The performance of the CFE method in estimating $\varphi_{\mathrm{Tx}}$ is also investigated in two measurements where the true $\varphi_{\mathrm{Tx}}=0^{\circ}$ and $25^{\circ}$ are specified, respectively. Figure 18 depicts the objective functions $\Lambda\left(\varphi_{\mathrm{Tx}} ; \mathbf{g}, \sigma_{s}, \mu_{m}, \sigma_{m}\right)$ calculated for the two measurements. It can be observed that the maxima of the objective functions, enclosed by ellipses in Figure 18, are located at $\varphi_{\mathrm{Tx}}=5^{\circ}$ and $23^{\circ}$ for the two cases, respectively. Since the antenna azimuth can be varied within the range significantly larger than that of the downtilt, the estimate with absolute estimation error less than $5^{\circ}$ may still be considered to be satisfactory in practice.

\section{Conclusions}

In this paper, a maximum-likelihood-based algorithm has been derived and used to estimate the attitude parameters, that is, the tilt directions of antennas at the BS based on the received signal strengths reported by multiple UEs located within the coverage of this BS. Depending on the availability of calibration campaigns, this algorithm can be utilized in either calibration-based estimation (CBE) or calibration-free estimation (CFE) of antenna attitude based on the empirical distributions of path loss, shadowing, and multipath fading. The CBE method relies on accurate path loss models and fading statistics extracted from calibration campaigns, while the CFE method makes use of only the general assumptions that the multipath and shadow fading gains follow the Rician and lognormal distributions, respectively. The performance of both methods was evaluated by using Monte-Carlo simulations, where a random-propagation-graph method has been applied for generating the channels impulse responses at multiple UEs' locations in an urban environment. The results demonstrated that the estimation accuracy for BS antenna attitude increases when more UEs with LoS connections with the BS antenna are considered. Experimental results obtained by processing measurement data demonstrated that the CBE method implemented by aggregating multiple objective functions provides more robust performance. Furthermore, without calibrations the CFE method still provides estimates of antenna downtilt and azimuth angle with errors tolerable from practical point of view.

\section{Conflict of Interests}

The authors declared that they have no conflict of interests to this work.

\section{Acknowledgments}

This work is jointly supported by Huawei Technology Company in the research project: Analysis and Verification of Channel Fingerprinting Characteristics in In-Service UMTS Networks (YBWL2010KJ010), Huawei innovation research project: High Dimensional MIMO Channel Estimation, Modeling and Measurement Combined with Antenna Status, the Science and Technology Commission of Shanghai Municipality project: System Design and Demo-Construction for Cooperative Networks of High-Efficiency 4G Wireless Communications in Urban Hot-Spot Environments (13510711000), the Key Program of National Natural Science Foundation of China (Grant no. 61331009), and the NSFC general project (Grant no. 61471268).

\section{References}

[1] K. A. Ali, H. S. Hassanein, and H. T. Mouftah, "QoS provisioning in WCDMA cellular networks through rate and coverage 
adaptation," in Proceedings of the 33rd IEEE Conference on Local Computer Networks (LCN '08), pp. 661-666, Montreal, Canada, October 2008.

[2] C. K. Ng, M. Ismail, B. M. Ali, S. Khatun, and S. S. Jamuar, "Impact of inter-cell interference on capacity in the joint multiple access (CDMA and SDMA) system," in Proceedings of the Asia-Pacific Conference on Communications, pp. 435-439, Western Australia, Australia, October 2005.

[3] T. S. Rappaport, Wireless Communications: Principles and Practice, Pearson Education, 2nd edition, 2002.

[4] T. Kaiser, Smart Antennas: State-of-the-Art, Hindawi Publishing Corporation, 2005.

[5] F. Kasem, A. Haskou, and Z. Dawy, "On antenna parameters self optimization in LTE cellular networks," in Proceedings of the $3 r d$ International Conference on Communications and Information Technology (ICCIT '13), pp. 44-48, Beirut, Lebanon, June 2013.

[6] I. Siomina, P. Värbrand, and Y. Di, "Automated optimization of service coverage and base station antenna configuration in UMTS networks," IEEE Wireless Communications, vol. 13, no. 6, pp. 16-25, 2006.

[7] Z. N. C. K.-M. Luk, Advanced Antennas for Radio Base Stations in Wireless Communications, McGraw-Hill, 2009.

[8] W. C. Y. Lee, Mobile Cellular Telecommunications Systems, McGraw-Hill, 1989.

[9] J. Niemela, T. Isotalo, J. Borkowski, and J. Lempiäinen, "Sensitivity of optimum downtilt angle for geographical traffic load distribution in WCDMA," in Proceedings of the 62nd IEEE Vehicular Technology Conference (VTC '05), vol. 2, pp. 12021206, Dallas, Tex, USA, September 2005.

[10] J. Lempiäinen and M. Manninen, UMTS Radio Network Planning, Optimization and QOS Management, Kluwer Academic Publishers, Boston, Mass, USA, 2003.

[11] 3GPP, “Tr 37.822 study on next-generation SON for UTRA and LTE,” Tech. Rep., 2014.

[12] I. Viering, M. Döttling, and A. Lobinger, "A mathematical perspective of self-optimizing wireless networks," in Proceedings of the IEEE International Conference on Communications (ICC '09), pp. 1-6, IEEE, Dresden, Germany, June 2009.

[13] W. Lee, "Cochannel interference reduction by using a notch in tilted antenna pattern," in Proceedings of the 35th IEEE Vehicular Technology Conference (VTC '85), pp. 67-72, Boulder, Colo, USA, May 1985.

[14] H.-S. Cho, Y.-I. Kim, and D. K. Sung, "Protection against cochannel interference from neighboring cells using down-tilting of antenna beams," in Proceedings of the 53rd IEEE Vehicular Technology Conference (VTC '01), Rhodes, Greece, May 2001.

[15] D. Sugimura, M. Arai, K. Sakaguchi, K. Araki, and T. Sotoyama, "A study on beam tilt angle of base station antennas for base station cooperation systems," in Proceedings of the IEEE 22nd International Symposium on Personal, Indoor and Mobile Radio Communications (PIMRC '11), pp. 2374-2378, September 2011.

[16] L. Thiele, T. Wirth, M. Schellmann, Y. Hadisusanto, and V. Jungnickel, "MU-MIMO with localized downlink base station cooperation and downtilted antennas," in Proceedings of the IEEE International Conference on Communications (ICC '09), Dresden, German, June 2009.

[17] M. Pettersen, L. E. Braten, and A. G. Spilling, "Automatic antenna tilt control for capacity enhancement in UMTS FDD," in Proceedings of the IEEE 60th Vehicular Technology Conference (VTC '04), vol. 1, pp. 280-284, September 2004.
[18] J.-S. Wu, J.-K. Chung, and C.-C. Wen, "Hot-spot traffic relief with a tilted antenna in CDMA cellular networks," IEEE Transactions on Vehicular Technology, vol. 47, no. 1, pp. 1-9, 1998.

[19] W. Jianhui and Y. Dongfeng, "Antenna downtilt performance in urban environments," in Proceedings of the IEEE Conference on Military Communications (MIL-COM '96), vol. 3, Mclean, Va, USA, October 1996.

[20] J. Niemelä, T. Isotalo, and J. Lempiäinen, "Optimum antenna downtilt angles for macrocellular WCDMA network," EURASIP Journal on Wireless Communications and Networking, vol. 2005, no. 5, Article ID 610942, 2005.

[21] U. J. Medbo, M. Riback, and J.-E. Berg, "Method and apparatus for improving the performance of a mobile radio communications system by adjusting antenna patterns," US Patent 8494588 B2, 2013.

[22] T. Jämsä, P. Kyösti, and K. Kusume, "Deliverable D1.2 initial channel models based on measurements," Tech. Rep., Mobile and Wireless Communications Enablers for the Twenty-Twenty Information Society (METIS), 2014.

[23] P. Guccione, A. M. Guarnieri, and M. Zonno, "Azimuth antenna maximum likelihood estimation by persistent point scatterers in SAR images," IEEE Transactions on Geoscience and Remote Sensing, vol. 52, no. 2, pp. 947-955, 2014.

[24] Y. K. C. Y. Inoue and Yokohama, "Base station apparatus and directivity control method in mobile commumication system," US Patent 8588835 B2, 2013.

[25] M. Francavilla, A. Schiavoni, P. Bertotto, and G. Richiardi, "Effect of the hand on cellular phone radiation," IEE Proceedings: Microwaves, Antennas and Propagation, vol. 148, no. 4, pp. 247-253, 2001.

[26] C.-M. Su, C.-H. Wu, K.-L. Wong, S.-H. Yeh, and C.-L. Tang, "User's hand effects on EMC internal GSM/DCS mobile phone antenna," in Proceedings of the IEEE Antennas and Propagation Society International Symposium (APS '06), pp. 2097-2100, IEEE, Albuquerque, NM, USA, July 2006.

[27] A. Goldsmith, Wireless Communication, Cambridge University Press, Cambridge, UK, 2005.

[28] G. L. Stüber, Principles of Mobile Communication, Kluwer Academic Publishers, New York, NY, USA, 2000.

[29] J. Salo, L. Vuokko, H. El-Sallabi, and P. Vainikainen, "Shadow fading revisited," in Proceedings of the 63rd IEEE Vehicular Technology Conference (VTC '06), vol. 6, pp. 2843-2847, Melbourne, Australia, May 2006.

[30] A. F. Molisch, Wireless Communication, Wiley Press, 2011.

[31] H. H. Xia, "A simplified analytical model for predicting path loss in urban and suburban environments," IEEE Transactions on Vehicular Technology, vol. 46, no. 4, pp. 1040-1046, 1997.

[32] L. J. Greenstein, D. G. Michelson, and V. Erceg, "Momentmethod estimation of the Ricean K-factor," IEEE Communications Letters, vol. 3, no. 6, pp. 175-176, 1999.

[33] J. Schroeder, S. Galler, K. Kyamakya, and K. Jobmann, "NLoS detection algorithms for ultra-wideband localization," in Proceedings of the Workshop on Positioning, Navigation and Communication (WPNC '07), Hanover, Germany, 2007.

[34] T. Pedersen and B. H. Fleury, "Radio channel modelling using stochastic propagation graphs," in Proceedings of the IEEE International Conference on Communications (ICC '07), pp. 2733-2738, June 2007.

[35] T. Pedersen, G. Steinbock, and B. H. Fleury, "Modeling of reverberant radio channels using propagation graphs," IEEE Transactions on Antennas and Propagation, vol. 60, no. 12, pp. 5978-5988, 2012. 
[36] L. Tian, X. Yin, X. Zhou, and Q. Zuo, "Spatial cross-correlation modeling for propagation channels in indoor distributed antenna systems," EURASIP Journal on Wireless Communications and Networking, vol. 2013, no. 1, article 183, 2013.

[37] T. Pedersen, G. Steinbock, and B. H. Fleury, "Modeling of outdoor-to-indoor radio channels via propagation graphs," in Proceedings of the 31th International Union of Radio Science General Assembly and Scientific Symposium (URSI GASS '14), pp. 1-4, Beijing, China, August 2014.

[38] R. Zhang, X. Lu, Z. Zhong, and L. Cai, "A study on spatialtemporal dynamics properties of indoor wireless channels," in Wireless Algorithms, Systems, and Applications: Proceedings of the 6th International Conference, WASA 2011, Chengdu, China, August 11-13, 2011, vol. 6843 of Lecture Notes in Computer Science, pp. 410-421, Springer, Berlin, Germany, 2011.

[39] L. Tian, X. Yin, Q. Zuo, J. Zhou, Z. Zhong, and S. X. Lu, "Channel modeling based on random propagation graphs for high speed railway scenarios," in Proceedings of the IEEE 23rd International Symposium on Personal, Indoor and Mobile Radio Communications (PIMRC '12), pp. 1746-1750, Sydney, Australia, September 2012.

[40] O. Souihli and T. Ohtsuki, "Benefits of rich scattering in MIMO channels: a graph-theoretical perspective," IEEE Communications Letters, vol. 17, no. 1, pp. 23-26, 2013.

[41] WINNER, "WINNER II channel models," IST-WINNER II Deliverable 1.1.2 v.1.2., 2007, http://www.ist-winner.org/deliverables.html.

[42] A. Maali, H. Mimoun, G. Baudoin, and A. Ouldali, "A new low complexity NLOS identification approach based on UWB energy detection," in Proceedings of the IEEE Radio and Wireless Symposium (RWS '09), pp. 675-678, San Diego, Calif, USA, January 2008. 

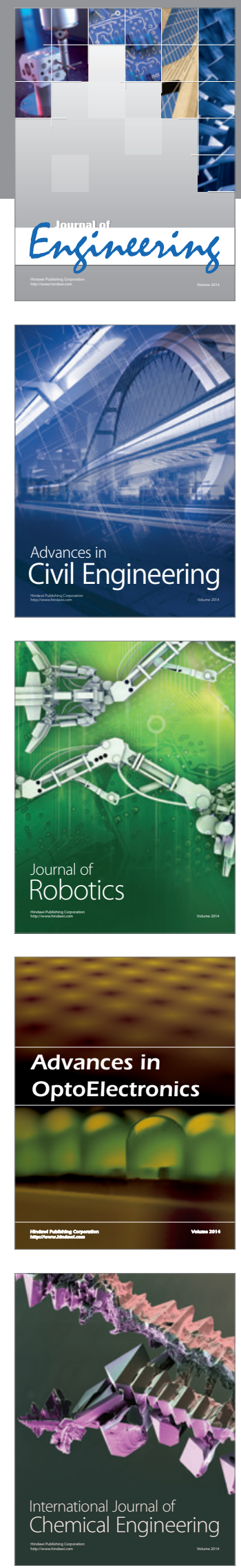

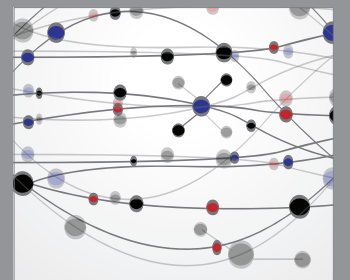

The Scientific World Journal
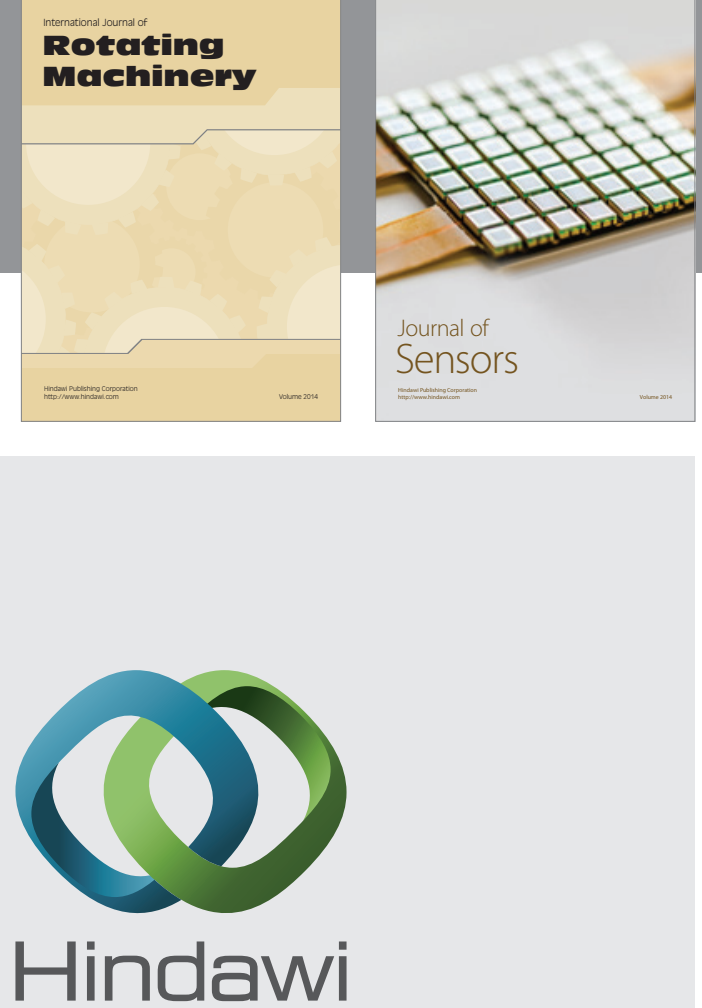

Submit your manuscripts at http://www.hindawi.com
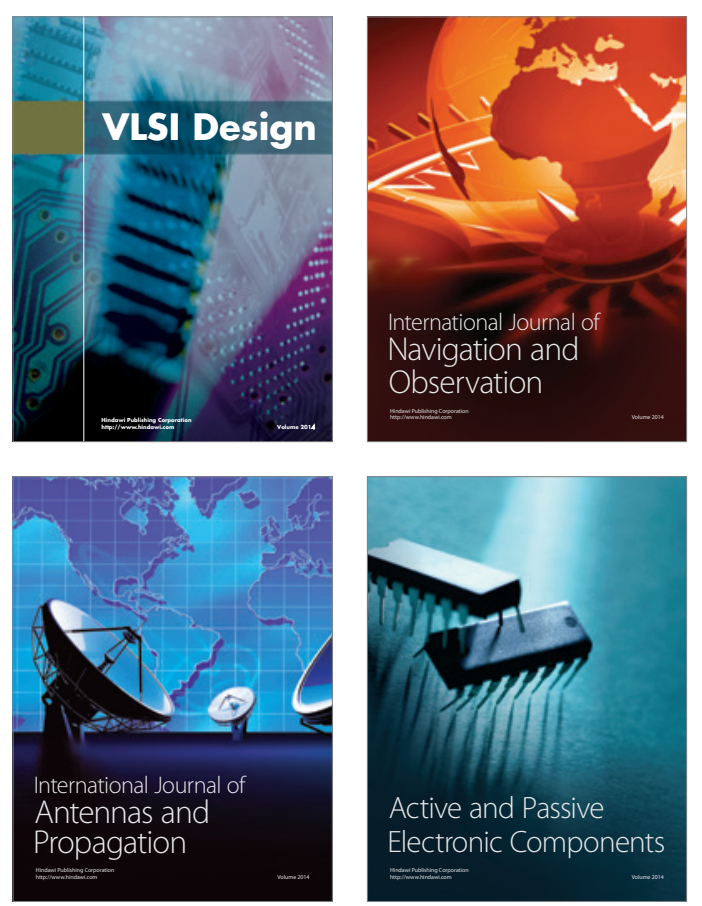
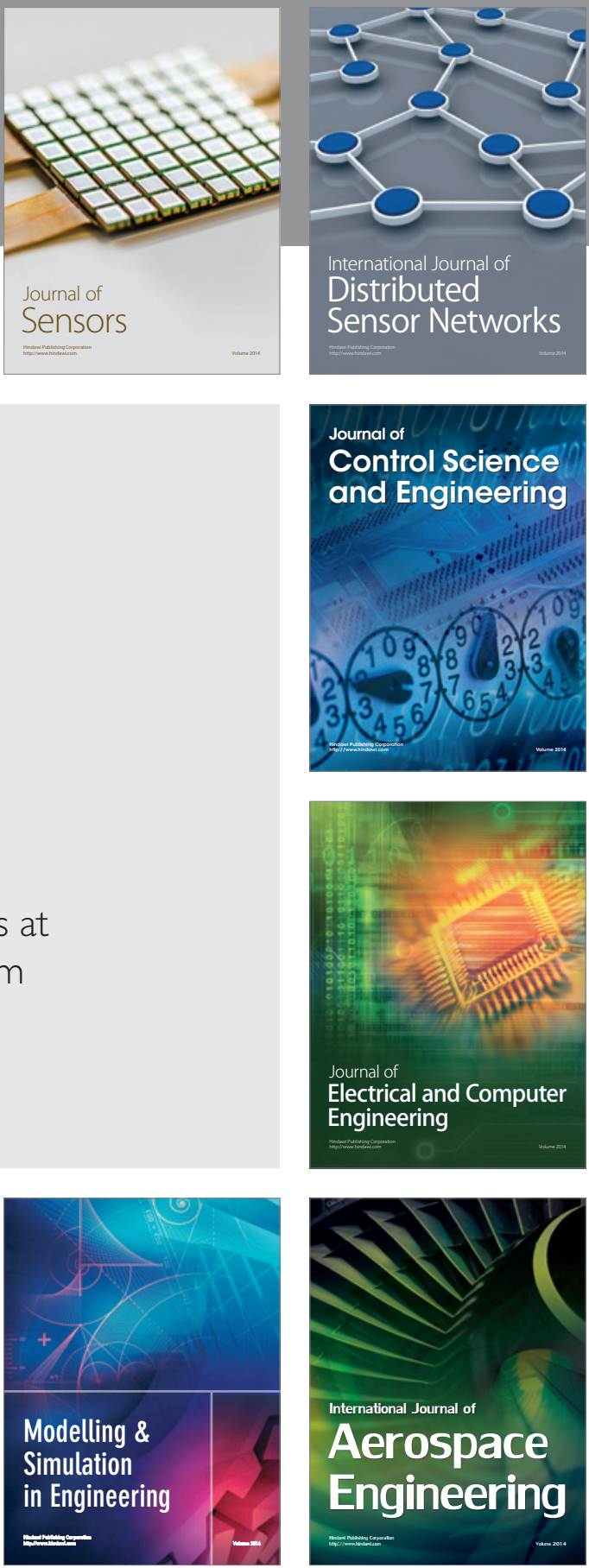

Journal of

Control Science

and Engineering
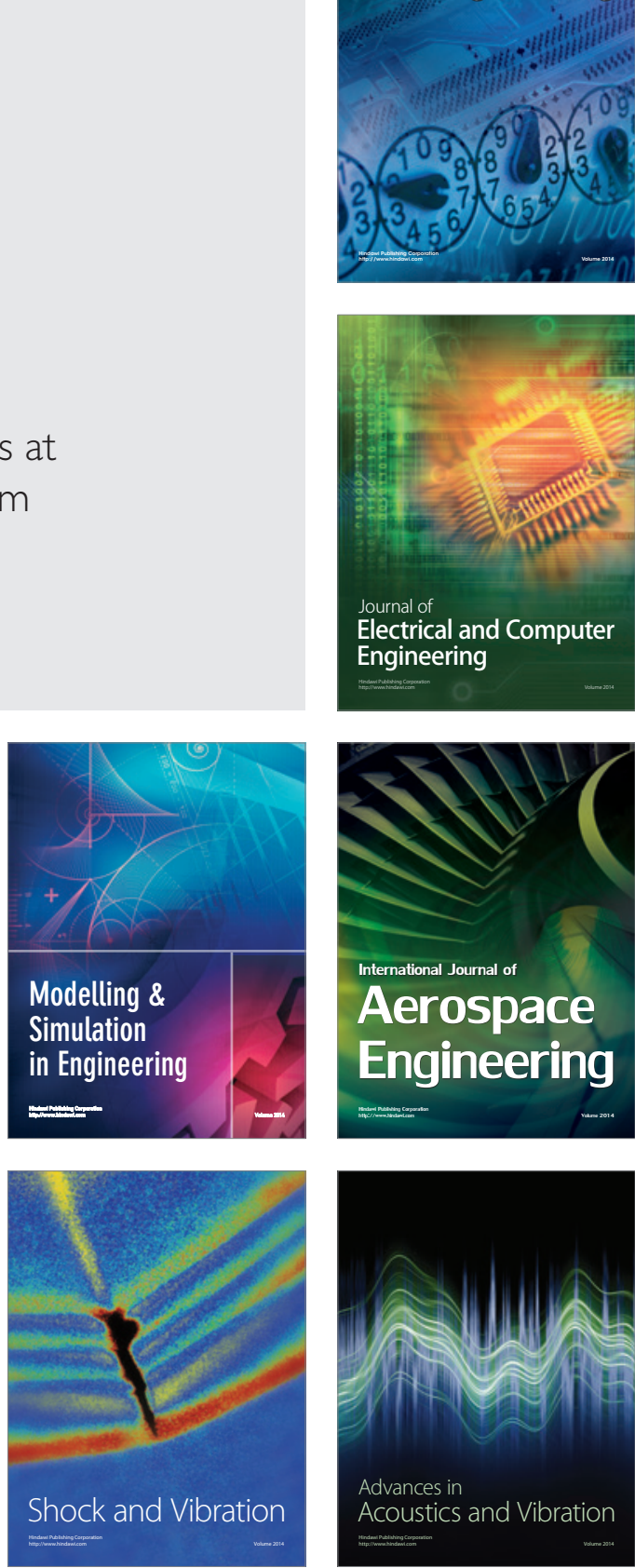